TRANSACTIONS OF THE

AMERICAN MATHEMATICAL SOCIETY

Volume 365, Number 11, November 2013, Pages 6037-6064

S 0002-9947(2013)05846-1

Article electronically published on June 13, 2013

\title{
DEMAZURE MODULES AND WEYL MODULES: THE TWISTED CURRENT CASE
}

\author{
GHISLAIN FOURIER AND DENIZ KUS
}

\begin{abstract}
We study finite-dimensional respresentations of twisted current algebras and show that any graded twisted Weyl module is isomorphic to level one Demazure modules for the twisted affine Kac-Moody algebra. Using the tensor product property of Demazure modules, we obtain, by analyzing the fundamental Weyl modules, dimension and character formulas. Moreover, we prove that graded twisted Weyl modules can be obtained by taking the associated graded modules of Weyl modules for the loop algebra, which implies that its dimension and classical character are independent of the support and depend only on its classical highest weight. These results were previously known for untwisted current algebras and are new for all twisted types.
\end{abstract}

\section{INTRODUCTION}

Weyl modules for loop algebras $\mathfrak{g} \otimes \mathbb{C}\left[t, t^{-1}\right]$, where $\mathfrak{g}$ is a simple complex Lie algebra, have received a lot of attention during the last two decades, starting with the analysis of finite-dimensional irreducible modules for quantum affine algebras (9), which are highest weight modules in a certain sense. It was natural to ask for maximal finite-dimensional modules with these highest weights since, contrary to the theory of simple complex Lie algebras, the category of finite-dimensional modules is not semisimple. In the same paper it was conjectured that the classical limit $q=1$ of these irreducible modules specialize to modules for the loop algebra satisfying some universal properties, the so-called local Weyl modules. In a series of papers $([1,6],[8$, [15], 24, [25]) the character and dimension of these Weyl modules were computed. In the proofs, these modules were identified with Weyl modules for the current algebra $\mathfrak{g} \otimes \mathbb{C}[t]$. Using the tensor product property ([9]) and some pullback maps, the study was reduced to analyzing graded Weyl modules for $\mathfrak{g} \otimes \mathbb{C}[t]$, where the grading is induced by the grading of $\mathbb{C}[t]$.

One major step in the analysis of the graded Weyl modules is their identification with level one Demazure modules for simply-laced algebras ([6], 15]). With the tensor product property for Demazure modules ([14]) and the computation for fundamental Weyl and Demazure modules ([6], 14]), the character and dimension formulas were proven. In the non-simply-laced case, Weyl modules admit a filtration by Demazure modules, and via this filtration, the dimension and character formula were proven $(25])$. One should mention that these results can also be deduced from the results in [1, 24, but there is no written proof so far in the literature.

Local Weyl modules for current and loop algebras can be parametrized by finitely supported functions from $\mathbb{C}\left(\right.$ resp. $\left.\mathbb{C}^{*}\right)$ to $P^{+}$, the set of dominant integral weights

Received by the editors September 12, 2011 and, in revised form, March 30, 2012.

2010 Mathematics Subject Classification. Primary 17B10; Secondary 17B65. 
for $\mathfrak{g}$. To each function one can associate a weight, which is the sum of all images, hence in $P^{+}$. To summarize the results above, the dimension and character of a local Weyl module are independent of the support of the parametrizing function and depend only on its weight. The graded local Weyl module of weight $\lambda$ is parametrized by the function of weight $\lambda$ with support only in the origin. We can also reformulate this result in terms of the global Weyl module, which is a projective module in a certain category and is in general infinite-dimensional. The results on local Weyl modules are equivalent to the statement that the global Weyl module is a free module for a certain commutative algebra $\mathbf{A}_{\lambda}$.

There are several ways to generalize the notion of local Weyl modules. By replacing $\mathbb{C}\left[t, t^{-1}\right]$ with a commutative, associative algebra ([12, 44) one can define local and global Weyl modules as before, obtain similar tensor product properties, but character and dimension formulas are known only in certain cases. Even for a case as simply looking as $\mathfrak{g}=\mathfrak{s l}_{2}$ and $\mathbb{C}\left[t_{1}, \ldots, t_{n}\right]$ with $n \geq 4$, there is no known dimension formula.

Another way of generalizing local Weyl modules is to look at twisted current and loop algebras. Given a complex simple Lie algebra $\mathfrak{g}$ and a commutative algebra $A$ $\left(=\mathbb{C}[t], \mathbb{C}\left[t, t^{-1}\right]\right)$, both equipped with the action of a finite group $\Gamma(\Gamma=\mathbb{Z} / m \mathbb{Z})$ by automorphism, one can extend this action to $\mathfrak{g} \otimes A$. The fixpoint Lie algebra $(\mathfrak{g} \otimes A)^{\Gamma}$ is called the twisted current algebra (resp. twisted loop algebra). The twisted current algebra is a subalgebra of the twisted affine Kac-Moody algebra associated to $\mathfrak{g}$, while the twisted loop is obtained by taking the quotient by the central element of the subalgebra without derivation [2].

Local Weyl modules for the twisted loop algebra were introduced and studied in [5]. It was proven that every Weyl module is the tensor product of Weyl modules located only in a single point. So to obtain dimension and character formulas it was sufficient to compute them for Weyl modules with support in a single point. The main theorem in [5] states that every Weyl module for the twisted loop algebra is isomorphic to the restriction of a Weyl module for the untwisted loop algebra. So all interesting information can be deduced from this isomorphism. In [16] the aforementioned global Weyl modules will be defined and studied for twisted loop algebras as well. It will be shown that the twisted global Weyl module is a submodule of the untwisted global Weyl module, viewed as a module for the twisted loop algebra by restriction. The results about the twisted local Weyl module translate again into the freeness of the twisted global Weyl module as a module for a certain commutative algebra $\mathbf{A}_{\lambda}^{\Gamma}$.

In 13 the notion of local Weyl modules was generalized to certain equivariant map algebras. Given $X$ an affine scheme and $\mathfrak{g}$ a finite-dimensional Lie algebra, both defined over an algebraically closed field and $\Gamma$ a finite group acting on $X$ and $\mathfrak{g}$ by automorphisms, the equivariant map algebra is the Lie algebra of equivariant maps from $X$ to $\mathfrak{g}$. In [13] several restrictions to this general case were assumed, and the group action on $X$ had to be free and abelian. But under these assumptions, the tensor product property was again proven. Furthermore, it was shown that every Weyl module for the equivariant map algebra is isomorphic to the restriction of a Weyl module for the algebra of maps from $X$ to $\mathfrak{g}$.

In this paper we are considering the gap in the computation of dimension and character formulas for local Weyl modules of twisted current algebras. For twisted and untwisted loop and current algebras, dimension formulas for all local Weyl 
modules are known except for graded local Weyl modules for the twisted current algebra. Let $\Gamma$ be the finite group of a non-trivial diagram automorphism of a simple Lie algebra $\mathfrak{g}$, so $\Gamma$ is of order 2 or 3 and $\mathfrak{g}$ of type $A, D, E$. In terms of equivariant map algebras, the affine scheme would be $X=\mathbb{C}$ and $\Gamma=\langle\xi\rangle$, where $\xi$ is the multiplication by a primitive 2 nd or 3rd root of unity. We see immediately that 0 is a fixpoint, so the group action is not free. In this setting, the results of 13] do not apply at the origin.

The goal of this paper is to compute a dimension and character formula for the local Weyl module located in 0 (the graded local Weyl module) of the twisted current algebra. The main tools are, as in [15] and 25], Demazure modules.

There are two cases to be considered. The first one is:

Theorem. Let $\mathfrak{g}$ not be of type $A_{2 l}$ and $\lambda \in P_{0}^{+}$. Then the local graded $(\mathfrak{g} \otimes \mathbb{C}[t])^{\Gamma_{-}}$ Weyl module $W^{\Gamma}(\lambda)$ is isomorphic to a Demazure module of level 1.

In the proof we will use the $\mathfrak{s l}_{2} \otimes \mathbb{C}[t]$ and the $\left(\mathfrak{s l}_{3} \otimes \mathbb{C}[t]\right)^{\Gamma}$ cases (proven in [9], 6], resp. Section 7). A tensor product property for Demazure modules was proven in [14, so to obtain a character formula for Weyl modules it is sufficient to determine the fundamental local Weyl modules, as done in Section 5 . Concluding, we were able to prove an analogous result to [5], [13, where the dimension of the local Weyl module does not depend on the support but only on the highest weight.

Theorem. For $\mathfrak{g}$ not of type $A_{2 l}$ and $\lambda \in P_{0}^{+}$, the local graded $(\mathfrak{g} \otimes \mathbb{C}[t])^{\Gamma}-$ Weyl module is isomorphic to the associated graded module of the restriction of a local Weyl module for $\mathfrak{g} \otimes \mathbb{C}\left[t, t^{-1}\right]$.

In the second case, we assume that $\mathfrak{g}$ is of type $A_{2 l}$; then the fixpoint algebra $\mathfrak{g}_{0}$ is of type $B_{l}$. With our methods here, one can only determine the local Weyl module for weights $\lambda$, where $\lambda\left(\alpha_{l}^{\vee}\right)$ is odd. In this case there is an identification with Demazure modules as before, so the graded local $(\mathfrak{g} \otimes \mathbb{C}[t])^{\Gamma}$-Weyl module is isomorphic to a Demazure module of level 1. Furthermore, we are able to show the following:

Theorem. Let $\lambda=\lambda_{1}+\lambda_{2} \in P_{0}^{+}$, where $\lambda_{2}\left(\alpha_{l}^{\vee}\right)$ is odd and $a \in \mathbb{C}^{*}$. Then

$$
W^{\Gamma}(\lambda) \cong \operatorname{gr}\left(W_{a}\left(\lambda_{1}\right) \otimes W^{\Gamma}\left(\lambda_{2}\right)\right),
$$

where $W_{a}\left(\lambda_{1}\right)$ is the local Weyl module for $\mathfrak{g} \otimes \mathbb{C}\left[t, t^{-1}\right]$, supported in a with highest weight $\lambda_{1}$.

In the case where $\lambda\left(\alpha_{l}^{\vee}\right)$ is even, the dimension and character of the local Weyl modules remains uncomputed and the identification with Demazure modules fails. Here we can state only a conjecture.

Conjecture. Let $\lambda \in P_{0}^{+}$. Then the graded local Weyl module is isomorphic to the associated graded module of the restriction of a local Weyl module for $\mathfrak{g} \otimes \mathbb{C}\left[t, t^{-1}\right]$. The dimension of a local Weyl module of highest weight $\lambda$ is independent of the support of the module.

The structure of the paper is as follows. In Section 2 are basics and notation for affine Kac-Moody algebras, recalled in Section 3 for twisted current algebras. In Section 4 Demazure and Weyl modules are defined. In Section 5 we identify Demazure modules with Weyl modules and determine the "smallest" Weyl modules. In Section [6] we show that every graded Weyl module of the twisted current algebra 
can be obtained by taking the associated graded module of the restriction of an untwisted loop module. In Section 7 the case $\mathfrak{g}=\mathfrak{s l}_{3}$ is treated separately, since it is used in some of the proofs of the other cases.

\section{The AfFine KaC-Moody Algebras}

2.1. Notation and basic results. In this section we fix the notation and the usual technical padding. Let $\mathfrak{g}=\mathfrak{g}(A)$ be a simple complex Lie algebra of rank $l$ associated to a Cartan matrix $A$ of finite type, denote $I=\{1, \ldots, l\}$. We fix a Cartan subalgebra $\mathfrak{h}$ in $\mathfrak{g}$ and a Borel subalgebra $\mathfrak{b} \supseteq \mathfrak{h}$. Denote $\Phi \subseteq \mathfrak{h}^{*}$ as the root system of $\mathfrak{g}$, and, corresponding to the choice of $\mathfrak{b}$, let $\Phi^{+}$be the set of positive roots and let $\Pi=\left\{\alpha_{1}, \ldots, \alpha_{l}\right\}$ be the corresponding basis of $\Phi$.

For a root $\beta \in \Phi$ let $\beta^{\vee} \in \mathfrak{h}$ be its coroot. The basis of the dual root system (also called the coroot system) $\Phi^{\vee} \subset \mathfrak{h}$ is denoted $\Pi^{\vee}=\left\{\alpha_{1}^{\vee}, \ldots, \alpha_{l}^{\vee}\right\}$. The Weyl group $W$ of $\Phi$ is generated by the simple reflections $s_{i}=s_{\alpha_{i}}$ associated to the simple roots.

Let $P=\bigoplus_{i=1}^{l} \mathbb{Z} \omega_{i}$ be the weight lattice of $\mathfrak{g}$ and let $P^{+}=\bigoplus_{i=1}^{l} \mathbb{Z}_{\geq 0} \omega_{i}$ be the subset of dominant weights. The group algebra of $P$ is denoted $\mathbb{Z}[P]$, and we write $\chi=\sum a_{\mu} e^{\mu}$ (finite sum, $\mu \in P, a_{\mu} \in \mathbb{Z}$ ) for an element in $\mathbb{Z}[P]$, where the embedding $P \hookrightarrow \mathbb{Z}[P]$ is defined by $\mu \mapsto e^{\mu}$. Further, we denote by $Q=\bigoplus_{i=1}^{l} \mathbb{Z} \alpha_{i}$ (respectively $Q^{+}=\bigoplus_{i=1}^{l} \mathbb{Z}_{\geq} \alpha_{i}$ ) the root (respectively positive root) lattice and let $\left\{x_{i}^{ \pm}, h_{i} \mid i \in I\right\}$ be a set of Chevalley generators of $\mathfrak{g}$.

Let $\widehat{\mathfrak{g}}$ be the affine Kac-Moody algebra (twisted or untwisted) corresponding to the Cartan matrix $\widehat{A}=\left(a_{i, j}\right)$. Note that if $\widehat{\mathfrak{g}}$ is an untwisted affine Kac-Moody algebra associated to $\mathfrak{g}$,

$$
\widehat{\mathfrak{g}}=\mathfrak{g} \otimes_{\mathbb{C}} \mathbb{C}\left[t, t^{-1}\right] \oplus \mathbb{C} c \oplus \mathbb{C} d .
$$

Here $d$ denotes the derivation $d=t \frac{d}{d t}$ and $c$ is the canonical central element. Recall that the Lie bracket is defined as

$\left[x \otimes t^{m}+\lambda c+\mu d, y \otimes t^{n}+\nu c+\eta d\right]=[x, y] \otimes t^{n+m}+\mu n y \otimes t^{n}+\eta m x \otimes t^{m}+m \delta_{m,-n}(x, y) c$.

We assume $\widehat{\mathfrak{g}}$ is arbitrary (possibly twisted), and we fix a Cartan subalgebra $\widehat{\mathfrak{h}}$ in $\widehat{\mathfrak{g}}$ and a Borel subalgebra $\widehat{\mathfrak{b}} \supseteq \widehat{\mathfrak{h}}, \Pi=\left\{\alpha_{0}, \ldots, \alpha_{l}\right\}$ the set of simple roots, and $\Pi^{\vee}=\left\{\alpha_{0}^{\vee}, \ldots, \alpha_{l}^{\vee}\right\}$ the set of simple coroots. Denote by $\widehat{\Phi}$ the root system of $\widehat{\mathfrak{g}}$ and let $\widehat{\Phi}^{+}$be the subset of positive roots. We denote by $\widehat{P}$ the weight lattice of $\widehat{\mathfrak{g}}$ and let $\widehat{P^{+}}$be the subset of dominant weights. The Weyl group $\widehat{W}$ of $\widehat{\Phi}$ is generated by the simple reflections $s_{i}=s_{\alpha_{i}}$ associated to the simple roots. Further, we fix vectors $w=\left(a_{0}, \ldots, a_{l}\right)^{t}, v=\left(a_{0}^{\vee}, \ldots, a_{l}^{\vee}\right)$ such that $v \widehat{A}=\widehat{A} w=0$. Here $v$ and $w$ are uniquely determind up to scalars. Then it is known that the center of $\widehat{\mathfrak{g}}$ is 1-dimensional and is spanned by the canonical central element

$$
c=\sum_{i=0}^{l} a_{i}^{\vee} \alpha_{i}^{\vee} .
$$

Define further

$$
\delta=\sum_{i=0}^{l} a_{i} \alpha_{i} ; \quad \theta=\delta-a_{0} \alpha_{0}
$$


and $d \in \widehat{\mathfrak{h}}$ which satisfies the following conditions:

$$
\alpha_{i}(d)=0, \text { for } i=1, \ldots, l ; \quad \alpha_{0}(d)=1 .
$$

Clearly the elements $\alpha_{0}^{\vee}, \ldots, \alpha_{l}^{\vee}, d$ form a basis of $\widehat{\mathfrak{h}}$. We have a non-degenerate symmetric bilinear form $\langle\cdot, \cdot\rangle$ on $\widehat{\mathfrak{h}}$ defined in ([19], Chapter 6$)$ :

$$
\begin{cases}\left\langle\alpha_{i}^{\vee}, \alpha_{j}^{\vee}\right\rangle=\frac{a_{j}}{a_{j}^{\vee}} a_{i, j}, & i, j=0, \ldots, \ell, \\ \left\langle\alpha_{i}^{\vee}, d\right\rangle=0, & i=1, \ldots, \ell, \\ \left\langle\alpha_{0}^{\vee}, d\right\rangle=\frac{a_{0}}{a_{0}^{\vee}}, & \langle d, d\rangle=0 .\end{cases}
$$

This $\widehat{W}$-invariant form induces a map

$$
\nu: \widehat{\mathfrak{h}} \longrightarrow \widehat{\mathfrak{h}}^{*}, \quad \nu(h):\left\{\begin{aligned}
\widehat{\mathfrak{h}} & \rightarrow \mathbb{C}, \\
h^{\prime} & \mapsto\left\langle h, h^{\prime}\right\rangle .
\end{aligned}\right.
$$

With the notation as above it follows for $i=0, \ldots, l$ that

$$
\nu\left(\alpha_{i}^{\vee}\right)=\frac{a_{i}}{a_{i}^{\vee}} \alpha_{i} .
$$

Let $\Lambda_{0}, \ldots, \Lambda_{l}$ be the fundamental weights in $\widehat{P^{+}}$. Then for $i=1, \ldots, l$ we have

$$
\Lambda_{i}=\omega_{i}+\frac{a_{i}^{\vee}}{a_{0}^{\vee}} \Lambda_{0}
$$

With this we have $\widehat{P}=\sum_{i=0}^{l} \mathbb{Z} \Lambda_{i}+\mathbb{Z}\left(\delta / a_{0}\right)$ and $\widehat{P^{+}}=\sum_{i=0}^{l} \mathbb{Z}_{\geq 0} \Lambda_{i}+\mathbb{Z}\left(\delta / a_{0}\right)$.

2.2. Realization of twisted affine algebras. In this paper we are mainly interested in twisted affine Kac-Moody algebras, which can be realized as fixed point subalgebras of so-called twisted graph automorphisms. Let $\mathfrak{g}$ be a finite-dimensional simple Lie algebra and $\sigma: \mathfrak{g} \rightarrow \mathfrak{g}$ be a graph automorphism of order $m$. In particular,

$$
m= \begin{cases}2, & \text { if } \mathfrak{g} \text { of type } A_{2 l}, A_{2 l-1}, D_{l+1} \text { or } E_{6}, \\ 3, & \text { if } \mathfrak{g} \text { is of type } D_{4} .\end{cases}
$$

Let $\xi$ be a primitive $m$ th root of unity. Then it is well known that there exists a decomposition of $\mathfrak{g}$ into eigenspaces. We obtain

$$
\mathfrak{g}=\mathfrak{g}_{0} \oplus \cdots \oplus \mathfrak{g}_{m-1},
$$

whereby $\mathfrak{g}_{j}=\left\{x \in \mathfrak{g} \mid \sigma(x)=\xi^{j} x\right\}, j=0, \ldots, m-1$. The fixed point algebra $\mathfrak{g}_{0}$ is again a simple complex Lie algebra of type $C_{l}, B_{l}, F_{4}$ or $G_{2}$, and the eigenspaces are irreducible $\mathfrak{g}_{0}$-modules.

Remark 2.1. Let $\mathfrak{a}$ be a subalgebra of $\mathfrak{g}$ such that $\sigma(\mathfrak{a})=\mathfrak{a}$. Then we get an analogue decomposition

$$
\mathfrak{a}=\mathfrak{a}_{0} \oplus \cdots \oplus \mathfrak{a}_{m-1} .
$$

So if $\mathfrak{g}=\mathfrak{n} \oplus \mathfrak{h} \oplus \mathfrak{n}_{-}$is a triangular decomposition of $\mathfrak{g}$, we obtain

$$
\mathfrak{g}_{j}=\mathfrak{n}_{j} \oplus \mathfrak{h}_{j} \oplus\left(\mathfrak{n}_{-}\right)_{j} \text { for all } 0 \leq j \leq m-1 \text {. }
$$

Now we can extend $\sigma$ to an automorphism of the corresponding untwisted affine algebra given by

$$
\begin{gathered}
\sigma\left(x \otimes t^{i}\right)=\xi^{-i} \sigma(x) \otimes t^{i} \quad \text { for } x \in \mathfrak{g}, \\
\sigma(c)=c ; \quad \sigma(d)=d .
\end{gathered}
$$


The twisted affine algebra is realized as the fixed point subalgebra

$$
\begin{gathered}
\widehat{\mathfrak{g}} \cong \bigoplus_{k \in \mathbb{Z}}\left(\mathfrak{g}_{0} \otimes t^{m k}\right) \oplus \cdots \oplus \bigoplus_{k \in \mathbb{Z}}\left(\mathfrak{g}_{m-1} \otimes t^{m k+(m-1)}\right) \oplus \mathbb{C} c \oplus \mathbb{C} d \\
=\bigoplus_{j=0}^{m-1} \bigoplus_{k \in \mathbb{Z}}\left(\mathfrak{g}_{j} \otimes t^{m k+j}\right) \oplus \mathbb{C} c \oplus \mathbb{C} d .
\end{gathered}
$$

Using the above notation we can conclude

$$
\widehat{\mathfrak{h}}=\mathfrak{h}_{0} \oplus(\mathbb{C} c+\mathbb{C} d), \quad \widehat{\mathfrak{h}}^{*}=\left(\mathfrak{h}_{0}\right)^{*} \oplus\left(\mathbb{C} \delta+\mathbb{C} \Lambda_{0}\right) .
$$

\begin{tabular}{|c|c|c|c|c|c|c|}
\hline$m$ & $\mathfrak{g}$ & $\mathfrak{g}_{0}$ & $\widehat{\mathfrak{g}}$ & $\mathfrak{g}_{1}$ & $\mathfrak{g}_{2}$ & Dynkin diagram of $\widehat{\mathfrak{g}}$ \\
\hline 2 & $A_{2}$ & $A_{1}$ & $A_{2}^{(2)}$ & $V\left(4 \omega_{1}\right)$ & / & $\circ \equiv$ \\
\hline 2 & $A_{2 l}, l \geq 2$ & $B_{l}$ & $A_{2 l}^{(2)}$ & $V\left(2 \omega_{1}\right)$ & / & 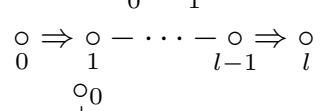 \\
\hline 2 & $A_{2 l-1}, l \geq 2$ & $C_{l}$ & $A_{2 l-1}^{(2)}$ & $V\left(\omega_{2}\right)$ & / & $\underset{1}{\circ}-\stackrel{o}{2}_{2}^{\mid}-\cdots-o_{l-1}^{\circ} \Leftarrow o_{l}^{\circ}$ \\
\hline 2 & $D_{l+1}, l \geq 3$ & $B_{l}$ & $D_{l+1}^{(2)}$ & $V\left(\omega_{1}\right)$ & / & $\stackrel{\circ}{\circ} \underset{1}{\circ}-\cdots-\underset{l-1}{\circ} \Rightarrow \underset{l}{\circ}$ \\
\hline 2 & $E_{6}$ & $F_{4}$ & $E_{6}^{(2)}$ & $V\left(\omega_{1}\right)$ & / & $\stackrel{\circ}{\circ}-\stackrel{\circ}{\circ}-{ }_{2}^{\circ} \Leftarrow{ }_{3}^{\circ}-\bigcirc_{4}^{\circ}$ \\
\hline 3 & $D_{4}$ & $G_{2}$ & $D_{4}^{(3)}$ & $V\left(\omega_{2}\right)$ & $V\left(\omega_{2}\right)$ & $\begin{array}{c}\circ \\
1\end{array} \Rightarrow \begin{array}{c}\circ \\
2\end{array}-\circ 0$ \\
\hline
\end{tabular}

We have the following table, which describes the various possibilities for $\mathfrak{g}, \mathfrak{g}_{0}, \widehat{\mathfrak{g}}$ and the eigenspaces $\mathfrak{g}_{1}, \mathfrak{g}_{2}$ :

We put a "0" on (almost) everything related to $\mathfrak{g}_{0}$, e.g. denote by $\Phi_{0} \subseteq\left(\mathfrak{h}_{0}\right)^{*}$ the root system of $\mathfrak{g}_{0}$. The recently defined element $\delta$ is the imaginary root in $\widehat{\Phi}^{+}$, and $\theta$ is the highest short root of the root system of $\mathfrak{g}_{0}$ if $\widehat{A}$ is of type $A_{2 l-1}^{(2)}, D_{l+1}^{(2)}, E_{6}^{(2)}, D_{4}^{(3)}$. In the remaining twisted cases $\theta-\alpha_{1}$ is the highest root of the root system of $\mathfrak{g}_{0}$. For more details we refer the reader to [19], 2].

Remark 2.2. The untwisted Kac-Moody algebras $\widehat{\mathfrak{g}}=\mathfrak{g} \otimes_{\mathbb{C}} \mathbb{C}\left[t, t^{-1}\right] \oplus \mathbb{C} c \oplus \mathbb{C} d$ can also be realized as fixed point algebras for any automorphism of order 1 . We have $\mathfrak{g}_{0}=\mathfrak{g}$, and the eigenspaces are the zerospaces. In this case $\theta$ is the highest root of $\mathfrak{g}$.

2.3. The extended affine Weyl group. Now we give a description of the Weyl group $\widehat{W}$ of the affine Kac-Moody algebra $\widehat{\mathfrak{g}}$. The Weyl group is generated by fundamental reflections $s_{0}, \ldots, s_{l}$, which act on $\widehat{\mathfrak{h}}^{*}$ by

$$
s_{i}(\lambda)=\lambda-\lambda\left(\alpha_{i}^{\vee}\right) \alpha_{i}, \quad \lambda \in \widehat{\mathfrak{h}}^{*} .
$$

Since $\delta\left(\alpha_{i}^{\vee}\right)=0$ for all $i=0, \ldots, l$, the Weyl group $\widehat{W}$ fixes $\delta$. Another well-known description of the affine Weyl group is the following. Let $W_{0}$ be the subgroup of $\widehat{W}$ generated by $s_{1}, \ldots, s_{l}$, i.e. $W_{0}$ can be identified with the Weyl group of the Lie algebra $\mathfrak{g}_{0}$, since $W_{0}$ operates trivially on $\left(\mathbb{C} \delta+\mathbb{C} \Lambda_{0}\right)$. Further, let

$$
M=\sum_{i=1}^{l} \mathbb{Z} \alpha_{i} \quad \text { if } \widehat{A} \text { symmetric or } m>a_{0}
$$




$$
M=\nu\left(\sum_{i=1}^{l} \mathbb{Z} \alpha_{i}^{\vee}\right) \quad \text { otherwise. }
$$

For an element $\mu \in M$, let $t_{\mu}$ be the following endomorphism of the vector space $\widehat{\mathfrak{h}}^{*}$ :

$$
\Lambda=\lambda+b \Lambda_{0}+r \delta \mapsto t_{\mu}(\Lambda)=\Lambda+\Lambda(c) \mu-\left(\langle\Lambda, \mu\rangle+\frac{1}{2}\langle\mu, \mu\rangle \Lambda(c)\right) \delta .
$$

Obviously we have $t_{\mu} \circ t_{\mu^{\prime}}=t_{\mu+\mu^{\prime}}$; denote $t_{M}$ as the abelian group consisting of the elements $t_{\mu}, \mu \in M$. Then $\widehat{W}$ is the semidirect product $\widehat{W}=W_{0} \ltimes t_{M}$.

The extended affine Weyl group $\widehat{W}^{\text {ext }}$ is the semidirect product $\widehat{W}^{\text {ext }}=\widehat{W} \ltimes t_{L}$, where $L=\nu\left(\bigoplus_{i=1}^{l} \mathbb{Z} \omega_{i}^{\vee}\right)$ is the image of the coweight lattice. The action of an element $t_{\mu}, \mu \in L$, is defined as above in (2.5). Let $\Sigma$ be the subgroup of $\widehat{W}^{\text {ext }}$ stabilizing the dominant Weyl chamber $\widehat{C}$ :

$$
\Sigma=\left\{\sigma \in \widehat{W}^{e x t} \mid \sigma(\widehat{C})=\widehat{C}\right\} .
$$

Then $\Sigma$ provides a complete system of coset representatives of $\widehat{W}^{e x t} \widehat{W}$, so we can in fact write $\widehat{W}^{e x t}=\Sigma \ltimes \widehat{W}$.

The elements $\sigma \in \Sigma$ are all of the form

$$
\sigma=\tau_{i} t_{-\nu\left(\omega_{i}^{\vee}\right)}=\tau_{i} t_{-\omega_{i}},
$$

where $\omega_{i}^{\vee}$ is a miniscule fundamental coweight. Further, set $\tau_{i}=w_{0} w_{0, i}$, where $w_{0}$ is the longest word in $W_{0}$ and $w_{0, i}$ is the longest word in $W_{\omega_{i}}$, the stabilizer of $\omega_{i}$ in $W_{0}$.

2.4. Weight space decomposition and roots. Remember that the Borel subalgebra for the twisted case is given by

$$
\begin{gathered}
\widehat{\mathfrak{b}}=\left(\left(\mathfrak{h}_{0} \oplus \mathfrak{n}_{0}\right) \otimes 1\right) \oplus \bigoplus_{k \in \mathbb{N}_{>0}}\left(\mathfrak{g}_{0} \otimes t^{m k}\right) \oplus \bigoplus_{k \in \mathbb{N}}\left(\mathfrak{g}_{1} \otimes t^{m k+1}\right) \\
\oplus \cdots \oplus \bigoplus_{k \in \mathbb{N}}\left(\mathfrak{g}_{m-1} \otimes t^{m k+(m-1)}\right) \oplus \mathbb{C} c \oplus \mathbb{C} d
\end{gathered}
$$

Furthermore, we remember that $\mathfrak{g}_{j}$ is an irreducible $\mathfrak{g}_{0}$-module for all $j$, so one can obtain the following weight space decomposition:

$$
\mathfrak{g}_{j}=\bigoplus_{\alpha \in\left(\mathfrak{h}_{0}\right)^{*}}\left(\mathfrak{g}_{j}\right)_{\alpha}
$$

Proposition 2.3. $\mathfrak{h}_{j}=\left(\mathfrak{g}_{j}\right)_{0} ; \quad\left(\mathfrak{n}_{-}\right)_{j}=\bigoplus_{\alpha \in \Phi^{-} \mid \mathfrak{h}_{0}}\left(\mathfrak{g}_{j}\right)_{\alpha} ; \quad \mathfrak{n}_{j}=\bigoplus_{\alpha \in \Phi^{+} \mid \mathfrak{h}_{0}}\left(\mathfrak{g}_{j}\right)_{\alpha}$, $0 \leq j \leq m-1$.

Let $\left(\Phi_{0}\right)_{s}$ be the set of short roots and $\left(\Phi_{0}\right)_{l}$ be the set of long roots of $\mathfrak{g}_{0}$ and $\Phi_{j}=\left\{\alpha \in\left(\mathfrak{h}_{0}\right)^{*} \mid\left(\mathfrak{g}_{j}\right)_{\alpha} \neq 0\right\}-\{0\}$. Then we get

$$
\mathfrak{g}_{j}=\mathfrak{h}_{j} \oplus \bigoplus_{\alpha \in \Phi_{j}}\left(\mathfrak{g}_{j}\right)_{\alpha}=\mathfrak{h}_{j} \oplus \bigoplus_{\alpha \in \Phi_{j}^{+}}\left(\mathfrak{g}_{j}\right)_{\alpha} \oplus \bigoplus_{\alpha \in \Phi_{j}^{-}}\left(\mathfrak{g}_{j}\right)_{\alpha},
$$


whereby $\operatorname{dim}\left(\mathfrak{g}_{j}\right)_{\alpha}=1$ for all $\alpha \in \Phi_{j}$, hence $\left(\mathfrak{g}_{j}\right)_{ \pm \alpha}=\mathbb{C} X_{\alpha, j}^{ \pm}$for $\alpha \in \Phi_{j}^{+}$, and we have the following table [2]:

\begin{tabular}{|c|c|c|c|c|}
\hline $\mathfrak{g}$ & $\mathfrak{g}_{0}$ & $\Phi_{1}$ & $\Phi_{2}$ & Dynkin diagram of $\mathfrak{g}_{0}$ \\
\hline$\overline{\overline{A_{2}}}$ & 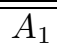 & 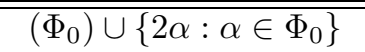 & $\overline{7 /}$ & $\circ$ \\
\hline$A_{2 l}, l \geq 2$ & $B_{l}$ & $\left(\Phi_{0}\right) \cup\left\{2 \alpha: \alpha \in\left(\Phi_{0}\right)_{s}\right\}$ & / & $\circ-\circ-\cdots-\circ \Rightarrow \circ$ \\
\hline$A_{2 l-1}, l \geq 2$ & $C_{l}$ & $\left(\Phi_{0}\right)_{s}$ & / & $\stackrel{\circ}{\circ}-{ }_{2}^{\circ}-\cdots-\underset{l-1}{\circ} \Leftarrow \circ$ \\
\hline$D_{l+1}, l \geq 3$ & $B_{l}$ & $\left(\Phi_{0}\right)_{s}$ & / & $\stackrel{\circ}{\circ}-\stackrel{\circ}{\circ}-\cdots-\stackrel{\circ}{\circ} \Rightarrow \stackrel{\circ}{\circ}$ \\
\hline$E_{6}$ & $F_{4}$ & $\left(\Phi_{0}\right)_{s}$ & 1 & $0-0 \Leftarrow 0-0$ \\
\hline$D_{4}$ & $G_{2}$ & $\left(\Phi_{0}\right)_{s}$ & $\left(\Phi_{0}\right)_{s}$ & $\circ \Rightarrow$ \\
\hline
\end{tabular}

\section{The twisted CURRent Algebra $\mathfrak{C}(\widehat{\mathfrak{g}})$}

In this section we will define the twisted current algebra $\mathfrak{C}(\widehat{\mathfrak{g}})$ and certain subalgebras which will be needed in the following sections. The main object of this paper will be

$$
\mathfrak{C}(\widehat{\mathfrak{g}}):=\bigoplus_{j=0}^{m-1} \bigoplus_{k \geq 0}\left(\mathfrak{g}_{j} \otimes t^{m k+j}\right) .
$$

The algebra $\mathfrak{C}(\widehat{\mathfrak{g}})$ can be realized by taking the fixpoints under the group of automorphisms $\Gamma$ restricted to the current algebra, in detail $(\mathfrak{g} \otimes \mathbb{C}[t])^{\Gamma} \cong \mathfrak{C}(\widehat{\mathfrak{g}})$. Hence it is called the twisted current algebra.

In order to give an explicit basis of $\mathfrak{C}(\widehat{\mathfrak{g}})$ we use the embedding $\mathfrak{g}_{j} \hookrightarrow \mathfrak{g}$ for all $0 \leq j \leq m-1$ so that we can realize the generators of the weight spaces $\left(\mathfrak{g}_{j}\right)_{ \pm \alpha}$ as elements in $\mathfrak{g}$. This is already described in [19], 2] and [5] if $\alpha$ is a simple root and can be continued to arbitrary $\alpha \in \Phi_{0}$ : Let $\left(\widetilde{\alpha_{1}}, \ldots, \widetilde{\alpha_{m}}\right)$ be an $m$-element orbit of $\sigma$ on $\Phi$ and $x_{\widetilde{\alpha}_{i}}^{ \pm} \in \mathfrak{g}$ be root vectors such that $\sigma\left(x_{\widetilde{\alpha_{i}}}^{ \pm}\right)=x_{\widetilde{\alpha_{j}}}^{ \pm}$, where $j \equiv i+1 \bmod m$. Then we obtain

$$
\left(\sum_{i=0}^{m-1}\left(\xi^{i}\right)^{j} x_{\sigma^{i}\left(\widetilde{\alpha_{1}}\right)}^{ \pm}\right) \in\left(\mathfrak{g}_{j}\right)_{ \pm \widetilde{\alpha_{1} \mid \mathfrak{h}_{0}}}, 0 \leq j \leq m-1 .
$$

In 2], Chapter 18.4, it is shown that the weight spaces of $\mathfrak{g}_{j}$ are spanned by such elements for all $m$-element orbits $\left(\widetilde{\alpha_{1}}, \ldots, \widetilde{\alpha_{m}}\right)$. So the weight spaces $\left(\mathfrak{g}_{j}\right)_{ \pm \alpha}$ can be described as follows: There has to be a root $\widetilde{\alpha}$ such that $\left.\widetilde{\alpha}\right|_{\mathfrak{h}_{0}}=\alpha$ and

$$
\mathbb{C} X_{\alpha, j}^{ \pm}=\mathbb{C}\left(\sum_{i=0}^{m-1}\left(\xi^{i}\right)^{j} x_{\sigma^{i}(\widetilde{\alpha})}^{ \pm}\right) .
$$

We further set

$$
\mathbb{C} h_{\alpha, j}=\mathbb{C}\left(\sum_{i=0}^{m-1}\left(\xi^{i}\right)^{j} h_{\sigma^{i}(\widetilde{\alpha})}\right) .
$$

At this point we have adapted our notation while we denote by $h_{\alpha, 0}$ the coroot of a root $\alpha \in \Phi_{0}$.

Lemma 3.1. Assume $\widehat{\mathfrak{g}}$ is of type $A_{2 l-1}^{(2)}, D_{l+1}^{(2)}, E_{6}^{(2)}$ or $D_{4}^{(3)}$. If $\alpha$ is a long root, then we get a canonical isomorphism

$$
\mathfrak{s l}_{2} \otimes \mathbb{C}[t] \cong\left\langle X_{\alpha, 0}^{ \pm} \otimes t^{m s}, h_{\alpha, 0} \otimes t^{m s} \mid s \in \mathbb{N}\right\rangle_{\mathbb{C}}=: \mathfrak{s l}_{2, \alpha} \otimes \mathbb{C}\left[t^{m}\right],
$$


and if $\alpha$ is short we have

$$
\mathfrak{s l}_{2} \otimes \mathbb{C}[t] \cong\left\langle X_{\alpha, j}^{ \pm} \otimes t^{m s+j}, h_{\alpha, j} \otimes t^{m s+j} \mid s \in \mathbb{N}, 0 \leq j \leq m-1\right\rangle_{\mathbb{C}}=: \mathfrak{s l}_{2, \alpha} \otimes \mathbb{C}[t] .
$$

Proof. Since the Lie algebra $\left\langle X_{\alpha, 0}^{ \pm}, h_{\alpha, 0}\right\rangle_{\mathbb{C}}$ is canonically isomorphic to $\mathfrak{s l}_{2}$, the first isomorphism is given by

$$
\begin{aligned}
x^{ \pm} \otimes t^{s} & \mapsto X_{\alpha, 0}^{ \pm} \otimes t^{m s}, \\
h \otimes t^{s} & \mapsto h_{\alpha, 0} \otimes t^{m s} .
\end{aligned}
$$

To verify the second isomorphism we define

$$
\begin{gathered}
x^{ \pm} \otimes t^{s} \mapsto X_{\alpha, j}^{ \pm} \otimes t^{s}, \text { if } s \equiv j \bmod m, \\
h \otimes t^{s} \mapsto h_{\alpha, j} \otimes t^{s}, \text { if } s \equiv j \bmod m .
\end{gathered}
$$

To show that this map is a homomorphism of Lie algebras we need to check

$$
\left[X_{\alpha, i_{1}}^{+}, X_{\alpha, i_{2}}^{-}\right]=h_{\alpha, i_{1}+i_{2}} \bmod m,\left[h_{\alpha, i_{2}}, X_{\alpha, i_{1}}^{ \pm}\right]= \pm 2 X_{\alpha, i_{1}+i_{2} \bmod m}^{ \pm}
$$

Since we require $\alpha$ to be a short root, we know that the weight space $\left(\mathfrak{g}_{j}\right)_{ \pm \alpha}, 0 \leq$ $j \leq m-1$, is non-zero, and therefore we can use the description in (3.1), (3.2) with $\widetilde{\alpha}$ such that $\sigma(\widetilde{\alpha}) \neq \widetilde{\alpha}$. More than this, a case by case consideration shows $\sigma^{j}(\widetilde{\alpha})\left(\sigma^{i}(\widetilde{\alpha})^{\vee}\right)=0$ and $\sigma^{j}(\widetilde{\alpha})-\sigma^{i}(\widetilde{\alpha})$ is not a root for $i \neq j$, e.g. in type $D_{l+1}^{(2)}$ we have for an arbitrary short root $\alpha_{i}+\cdots+\alpha_{l}$ of $B_{l}$, that $\widetilde{\alpha}=\alpha_{i}+\cdots+\alpha_{l}$ and therefore $\sigma(\widetilde{\alpha})-\widetilde{\alpha}=\alpha_{l+1}-\alpha_{l}$ is not a root and $\sigma(\widetilde{\alpha})\left(\widetilde{\alpha}^{\vee}\right)=\widetilde{\alpha}\left(\sigma(\widetilde{\alpha})^{\vee}\right)=0$. The proof in the other cases is similar. We set $X_{\alpha, j}^{ \pm}=\left(\sum_{i=0}^{m-1}\left(\xi^{i}\right)^{j} x_{\sigma^{i}(\widetilde{\alpha})}^{ \pm}\right), h_{\alpha, j}=$ $\left(\sum_{i=0}^{m-1}\left(\xi^{i}\right)^{j} h_{\sigma^{i}(\widetilde{\alpha})}\right)$. The required equations in (3.3) are now immediate.

If $\widehat{\mathfrak{g}}$ is of type $A_{2 l}^{(2)}$, we obtain a similar result.

Lemma 3.2. Assume $\widehat{\mathfrak{g}}$ is of type $A_{2 l}^{(2)}$ and $\alpha$ is a long root. Then we get a canonical isomorphism

$$
\mathfrak{s l}_{2} \otimes \mathbb{C}[t] \cong\left\langle X_{\alpha, j}^{ \pm} \otimes t^{m s+j}, h_{\alpha, j} \otimes t^{m s+j} \mid s \in \mathbb{N}, 0 \leq j \leq m-1\right\rangle_{\mathbb{C}}=: \mathfrak{s l}_{2, \alpha} \otimes \mathbb{C}[t] .
$$

If $\alpha$ is a short root, then we get a canonical isomorphism

$$
\mathfrak{C}\left(A_{2}^{(2)}\right) \cong\left\langle X_{\alpha, j}^{ \pm} \otimes t^{m s+j}, X_{2 \alpha, 1}^{ \pm} \otimes t^{m s+1}, h_{\alpha, j} \otimes t^{m s+j} \mid s \in \mathbb{N}, 0 \leq j \leq m-1\right\rangle_{\mathbb{C}} .
$$

Proof. The proof of the first isomorphism is similar to Lemma 3.1, and to justify the second isomorphism we will demonstrate how to realize the elements $h_{\alpha, j}, X_{\alpha, j}^{ \pm}$, $X_{2 \alpha, 1}^{ \pm}$as elements in $A_{2 l}$. Let $\alpha=\alpha_{i}+\cdots+\alpha_{l}$ be an arbitrary short root of type $B_{l}$ and $\widetilde{\alpha}=\alpha_{i}+\cdots+\alpha_{l}$ be the root considered as a root in type $A_{2 l}$, i.e. the restriction to $\mathfrak{h}_{0}$ equals $\alpha$. It is easy to see that $\sigma(\widetilde{\alpha}) \neq \widetilde{\alpha}, \sigma(\widetilde{\alpha})-\widetilde{\alpha}$ is not a root of $A_{2 l}$ and continuing $\sigma(\widetilde{\alpha})\left(\widetilde{\alpha}^{\vee}\right)=\widetilde{\alpha}\left(\sigma(\widetilde{\alpha})^{\vee}\right)=-1$. We set

$$
\begin{gathered}
X_{\alpha, j}^{ \pm}=(\xi)^{j} \sqrt{2}\left(x_{\widetilde{\alpha}}^{ \pm}+\xi^{j} x_{\sigma(\widetilde{\alpha})}^{ \pm}\right) \in\left(\mathfrak{g}_{j}\right)_{ \pm \alpha}, \\
X_{2 \alpha, 1}^{ \pm}=\left[x_{\widetilde{\alpha}}^{ \pm}, x_{\sigma(\widetilde{\alpha})}^{ \pm}\right] \in\left(\mathfrak{g}_{1}\right)_{ \pm 2 \alpha}, \\
h_{\alpha, j}=2^{\delta_{0, j}}\left(h_{\widetilde{\alpha}}+\xi^{j} h_{\sigma(\widetilde{\alpha})}\right) .
\end{gathered}
$$

Now, knowing the embedding in $A_{2 l}$, it is straightforward to check the required relations. 
3.1. Filtration on $\mathfrak{C}(\widehat{\mathfrak{g}})$, the twisted current algebra. The Lie algebra $\mathfrak{C}(\widehat{\mathfrak{g}})$ has a natural grading and an associated natural filtration $F^{\bullet}(\mathfrak{C}(\widehat{\mathfrak{g}}))$, where $F^{s}(\mathfrak{C}(\widehat{\mathfrak{g}}))$ is defined to be the subspace of $\mathfrak{g}$-valued polynomials with degree smaller than or equal to $s$. One also has an induced filtration on the enveloping algebra $U(\mathfrak{C}(\widehat{\mathfrak{g}}))$ and therefore an induced filtration on arbitrary cyclic $U(\mathfrak{C}(\widehat{\mathfrak{g}}))$-modules $W$ with cyclic vector $w$. Denote by $W_{s}$ the subspace spanned by the vectors of the form g.w, where $g \in F^{s}(U(\mathfrak{C}(\widehat{\mathfrak{g}})))$, and denote the associated graded $\mathfrak{C}(\widehat{\mathfrak{g}})$-module by $\operatorname{gr}(W)$ :

$$
\operatorname{gr}(W)=\bigoplus_{i \geq 0} W_{i} / W_{i-1}, \quad \text { where } W_{-1}=0
$$

\section{Demazure modules and Weyl modules}

4.1. Definition of Demazure modules. For a dominant weight $\Lambda \in \widehat{P}^{+}$let $V(\Lambda)$ be the irreducible highest weight module of highest weight $\Lambda$. Given an element $w \in \widehat{W}$, fix a generator $v_{w(\Lambda)}$ of the line $V(\Lambda)_{w(\Lambda)}=\mathbb{C} v_{w(\Lambda)}$ of $\widehat{\mathfrak{h}}$-eigenvectors in $V(\Lambda)$ of weight $w(\Lambda)$.

Definition 4.1. The $U(\widehat{\mathfrak{b}})$-submodule $V_{w}(\Lambda)=U(\widehat{\mathfrak{b}}) \cdot v_{w(\Lambda)}$ generated by $v_{w(\Lambda)}$ is called the Demazure submodule of $V(\Lambda)$ associated to $w$.

Remark 4.2.

(1) Since $\widehat{\mathfrak{h}}$ acts by multiplication with a scalar on $v_{w(\Lambda)}$, the Demazure module $V_{w}(\Lambda)$ is a cyclic $U(\widehat{\mathfrak{n}})$-module generated by $v_{w(\Lambda)}$.

(2) The modules $V_{w}(\Lambda)$ are finite-dimensional although $V(\Lambda)$ is infinite-dimensional.

To associate more generally to every element $\sigma w \in \widehat{W}^{e x t}=\Sigma \ltimes \widehat{W}$ a Demazure module, recall that elements in $\Sigma$ correspond to automorphisms of the Dynkin diagram of $\widehat{\mathfrak{g}}$ and thus define an associated automorphism of $\widehat{\mathfrak{g}}$, also denoted $\sigma$. For a module $V$ of $\widehat{\mathfrak{g}}$ let $\sigma^{*}(V)$ be the module with the twisted action $g \circ v=\sigma^{-1}(g) v$. Then for the irreducible module of highest weight $\Lambda \in \widehat{P}^{+}$we get $\sigma^{*}(V(\Lambda))=$ $V(\sigma(\Lambda))$.

So for $\sigma w \in \widehat{W}^{e x t}=\Sigma \times \widehat{W}$ we set

$$
V_{\sigma w}(\Lambda):=V_{\sigma w \sigma^{-1}}(\sigma(\Lambda)) .
$$

We are mainly interested in $\mathfrak{g}_{0}$-stable Demazure modules. For $i \in I_{0}$ we have $X_{\alpha_{i}, 0}^{-} v_{w(\Lambda)}=0$ if and only if $w(\Lambda)\left(\alpha_{i}^{\vee}\right) \leq 0$. Consequently, we can see that $V_{w}(\Lambda)$ is $\mathfrak{g}_{0}$-stable if and only if $w(\Lambda)\left(\alpha_{i}^{\vee}\right) \leq 0$ for all $i \in I_{0}$. Assume that $\omega(\Lambda)=$ $-\lambda+k \Lambda_{0}+i \delta$; then $V_{w}(\Lambda)$ is stable under $\mathfrak{g}_{0}$ if and only if $\lambda \in P_{0}^{+}$. We define a set

$X=\left\{(\lambda, k, i) \in P_{0}^{+} \times\left(1 / a_{0}^{\vee}\right) \mathbb{Z}_{>0} \times\left(1 / a_{0}\right) \mathbb{Z} \mid \exists ! \Lambda \in \widehat{P}^{+}: w_{0}(\lambda)+k \Lambda_{0}+i \delta \in \widehat{W}(\Lambda)\right\}$, where $w_{0}$ is the longest word in $W_{0}$. Let $(\lambda, k, i) \in X$ and $\omega \in \widehat{W}$ such that $\omega(\Lambda)=w_{0}(\lambda)+k \Lambda_{0}+i \delta$. Then by the above computation we get the $\mathfrak{g}_{0}$-stability of the Demazure module $V_{w}(\Lambda)$ and we denote

$$
V_{w}(\Lambda)=D(k, \lambda)[i] .
$$

Remark 4.3.

(1) The $\mathfrak{g}_{0}$-stable Demazure modules are in fact $\mathfrak{C}(\widehat{\mathfrak{g}})$-modules. 
(2) For any $\Lambda \in \widehat{P}^{+}$and $i \in\left(1 / a_{0}\right) \mathbb{Z}$, we have $V(\Lambda) \cong V(\Lambda+i \delta)$ as $\mathfrak{C}(\widehat{\mathfrak{g}})$ modules. Therefore we get

$$
D(k, \lambda)[i] \cong D(k, \lambda)[i+n],
$$

which justifies the notation $D(k, \lambda)$ as a $\mathfrak{C}(\widehat{\mathfrak{g}})$-module.

Remark 4.4. Whenever we speak about $D(k, \lambda)$, we will assume that $(\lambda, k) \in X$. If $\widehat{\mathfrak{g}}$ is not of type $A_{2 l}^{(2)}(l \geq 1)$, the set $X$ is given by $X=P_{0}^{+} \times \mathbb{Z}_{>0} \times \mathbb{Z}$, and otherwise we have $P_{0}^{+} \times \mathbb{Z}_{>0} \times \mathbb{Z} \subsetneq X$.

4.2. Demazure character formula. Let $\beta$ be a real root of the root system $\widehat{\Phi}$. We define the Demazure operator:

$$
D_{\beta}: \mathbb{Z}[\widehat{P}] \rightarrow \mathbb{Z}[\widehat{P}], \quad D_{\beta}\left(e^{\lambda}\right)=\frac{e^{\lambda}-e^{s_{\beta}(\lambda)-\beta}}{1-e^{-\beta}} .
$$

\section{Lemma 4.5.}

(1) For $\lambda, \mu \in \widehat{P}$ we have

$$
D_{\beta}\left(e^{\lambda}\right)= \begin{cases}e^{\lambda}+e^{\lambda-\beta}+\cdots+e^{s_{\beta}(\lambda)} & \text { if }\left\langle\lambda, \beta^{\vee}\right\rangle \geq 0, \\ 0 & \text { if }\left\langle\lambda, \beta^{\vee}\right\rangle=-1, \\ -e^{\lambda+\beta}-e^{\lambda+2 \beta}-\cdots-e^{s_{\beta}(\lambda)-\beta} & \text { if }\left\langle\lambda, \beta^{\vee}\right\rangle \leq-2\end{cases}
$$

(2) Let $\chi, \eta \in \mathbb{Z}[\widehat{P}]$. If $D_{\beta}(\eta)=\eta$, then

$$
D_{\beta}(\chi \cdot \eta)=\eta \cdot\left(D_{\beta}(\chi)\right) .
$$

Proof. For (1) see [10, (1.5)-(1.8) and for (2) see [14, (2.2).

Since $D_{\alpha_{i}}\left(1-e^{\delta}\right)=\left(1-e^{\delta}\right)$ for all $i=0, \ldots, n$, (4.3) shows that the ideal $I_{\delta}=\left\langle\left(1-e^{\delta}\right)\right\rangle$ is stable under all Demazure operators $D_{\beta}$. Thus we obtain induced operators (we still use the same notation $D_{\beta}$ )

$$
D_{\beta}: \mathbb{Z}[\widehat{P}] / I_{\delta} \longrightarrow \mathbb{Z}[\widehat{P}] / I_{\delta}, \quad e^{\lambda}+I_{\delta} \mapsto D_{\beta}\left(e^{\lambda}\right)+I_{\delta} .
$$

In the following we denote by $D_{i}, i=0, \ldots, n$, the Demazure operator $D_{\alpha_{i}}$ corresponding to the simple root $\alpha_{i}$. Recall that for any reduced decomposition $w=s_{i_{1}} \cdots s_{i_{r}}$ of $w \in \widehat{W}$ the operator $D_{w}=D_{i_{1}} \cdots D_{i_{r}}$ is independent of the choice of the decomposition (see [21, Corollary 8.2.10). We have the following important theorem:

Theorem 4.6 ([21], Chapter VIII).

$$
\operatorname{Char} V_{w}(\Lambda)=D_{w}\left(e^{\Lambda}\right) .
$$

We will need the following elementary proposition:

Proposition 4.7. Let $\lambda_{1}^{\vee}, \lambda_{2}^{\vee}$ be two dominant coweights, and set $\lambda^{\vee}=\lambda_{1}^{\vee}+\lambda_{2}^{\vee}$. Then

(1) $D_{t_{-\nu(\lambda)}} D_{t_{-\nu\left(\lambda_{2}^{\vee}\right)}}=D_{t_{-\nu(\lambda \vee)}}$,

(2) $D_{t_{-\nu\left(\lambda_{1}^{\vee}\right)}} D_{\omega_{0}}=D_{t_{-\nu\left(\lambda_{1}^{\vee}\right)} \omega_{0}}$. 
4.3. Properties of Demazure modules. Since $V_{w}(\Lambda)=U(\widehat{\mathfrak{b}}) \cdot v_{w(\Lambda)}$, there exists an ideal $J \subseteq U(\widehat{\mathfrak{b}})$ such that $V_{w}(\Lambda) \cong U(\widehat{\mathfrak{b}}) / J$. So the Demazure module can be described by generators and relations, which was done in 23 . Here we give a reformulation for the twisted affine case:

Proposition $4.8([23])$. Let $\Lambda \in \widehat{P}^{+}$and let $w$ be an element of the affine Weyl group of $\widehat{\mathfrak{g}}$. The Demazure module $V_{w}(\Lambda)$ is as a $U(\widehat{\mathfrak{b}})$-module isomorphic to the cyclic module, generated by $v \neq 0$ with respect to the following relations.

For $\beta \in \Phi_{j}^{+}, 0 \leq j \leq m-1$ we have

$$
\begin{aligned}
& \left(X_{\beta, j}^{+} \otimes t^{m s+j}\right)^{k_{\beta}+1} . v=0, \\
& \quad \text { where } s \geq 0, \quad k_{\beta}=\max \left\{0,-\left\langle w(\Lambda),(\beta+(m s+j) \delta)^{\vee}\right\rangle\right\}, \\
& \left(X_{\beta, j}^{-} \otimes t^{m s+j}\right)^{k_{\beta}+1} . v=0, \\
& \quad \text { where } s>-\delta_{j,\{1, \ldots, m-1\}}, \quad k_{\beta}=\max \left\{0,-\left\langle w(\Lambda),(-\beta+(m s+j) \delta)^{\vee}\right\rangle\right\}, \\
& \left(h \otimes t^{m s+j}\right) . v=\delta_{j, 0} \delta_{s, 0} w(\Lambda)(h) v \quad \forall h \in \mathfrak{h}_{j}, \\
& \text { where } s \geq 0, \quad d . v=w(\Lambda)(d) . v, \quad c . v=w(\Lambda)(c) v .
\end{aligned}
$$

Corollary 4.9. As a module for $\mathfrak{C}(\widehat{\mathfrak{g}})$ the Demazure module $D(k, \lambda)$ is isomorphic to the cyclic $U(\mathfrak{C}(\widehat{\mathfrak{g}}))$-module generated by a vector $v \neq 0$ subject to the following relations:

For $\beta \in \Phi_{j}^{+}, 0 \leq j \leq m-1$ we have

$\mathfrak{n}_{j} \otimes t^{j} \mathbb{C}\left[t^{m}\right] . v=0$

$\left(X_{\beta, j}^{-} \otimes t^{m s+j}\right)^{k_{\beta}+1} . v=0, \quad$ where $s \geq 0, \quad k_{\beta}=\max \left\{0,\left\langle\lambda, \beta^{\vee}\right\rangle-\frac{2(m s+j)}{\langle\beta, \beta\rangle} k a_{0}^{\vee}\right\}$, $\left(h \otimes t^{m s+j}\right) . v=\delta_{j, 0} \delta_{s, 0} \lambda(h) v \quad \forall h \in \mathfrak{h}_{j}, \quad$ where $s \geq 0$.

Proof. The proof is similar to the one given in [15], Corollary 1.

Remark 4.10. Since the defining relations of $D(k, \lambda)$ respect the grading of $\mathfrak{C}(\widehat{\mathfrak{g}})$, $D(k, \lambda)$ is a graded module.

In 14 it was shown by using the Demazure operator that $D(k, \lambda)$ decomposes as a $\mathfrak{g}$ (resp. $\left.\mathfrak{g}_{0}\right)$ module into a tensor product of "smaller" Demazure modules. Here we give the result for the twisted affine case:

Theorem 4.11 ([14]). Let $\lambda^{\vee}=\lambda_{1}^{\vee}+\lambda_{2}^{\vee}+\cdots+\lambda_{r}^{\vee}$ be a sum of dominant coweights. Then for $m \geq 0$ we have an isomorphism of $\mathfrak{g}_{0}$-modules between the Demazure module $V_{-\lambda^{\vee}}\left(m \Lambda_{0}\right)$ and the tensor product of Demazure modules:

$$
V_{-\lambda \vee}\left(m \Lambda_{0}\right) \simeq V_{-\lambda_{1}^{\vee}}\left(m \Lambda_{0}\right) \otimes V_{-\lambda_{2}^{\vee}}\left(m \Lambda_{0}\right) \otimes \cdots \otimes V_{-\lambda_{r}^{\vee}}\left(m \Lambda_{0}\right) .
$$

Remark 4.12. This theorem holds for any special vertex $k$ of the twisted affine diagram.

4.4. Definition of local Weyl modules. The representation theory of twisted current algebras is particularly interesting because the category of finite-dimensional representation is not semisimple. It makes sense to ask for the "maximal" finitedimensional cyclic representations in this class, which leads to the definition of local Weyl modules. Please see [4] or [16] for the explanation of the term "local" in contrast to the term "global". 
Let $\lambda=\sum_{i=1}^{l} m_{i} \omega_{i} \in P_{0}^{+}$be a dominant integral weight for $\mathfrak{g}_{0}$. Then we define the local graded Weyl module $W^{\Gamma}(\lambda)$ in terms of generators and relations:

Definition 4.13. Let $\lambda=\sum_{i=1}^{l} m_{i} \omega_{i}$ be a dominant integral weight for $\mathfrak{g}_{0}$. Define $W^{\Gamma}(\lambda)$ to be the $U(\mathfrak{C}(\widehat{\mathfrak{g}}))$-module generated by an element $w_{\lambda}$ with the relations

$$
\begin{gathered}
\mathfrak{n}_{j} \otimes t^{j} \mathbb{C}\left[t^{m}\right] \cdot w_{\lambda}=0,0 \leq j \leq m-1, \\
\left(h \otimes t^{m s+j}\right) \cdot w_{\lambda}=\delta_{j, 0} \delta_{s, 0} \lambda(h) w_{\lambda} \quad \forall h \in \mathfrak{h}_{j}, \quad \text { where } s \geq 0, \\
\left(X_{\beta, 0}^{-} \otimes 1\right)^{\lambda\left(\beta^{\vee}\right)+1} w_{\lambda}=0, \text { for all positive roots } \beta \text { of } \mathfrak{g}_{0} .
\end{gathered}
$$

Remark 4.14. Note that the modules $W^{\Gamma}(\lambda)$ are graded modules since $U(\mathfrak{C}(\widehat{\mathfrak{g}}))$ is graded by the powers of $t$ and the defining relations are graded. Particularly, we have

$$
W^{\Gamma}(\lambda) \cong \bigoplus_{s \in \mathbb{Z}_{+}} W^{\Gamma}(\lambda)[s],
$$

where $W^{\Gamma}(\lambda)[s]$ is a $\mathfrak{g}_{0}$-module by identifying $\mathfrak{g}_{0}$ with $\mathfrak{g}_{0} \otimes 1 \subseteq \mathfrak{C}(\widehat{\mathfrak{g}})$.

\subsection{Properties of Weyl modules.}

\section{Proposition 4.15.}

(1) We have

$$
W^{\Gamma}(\lambda)=\bigoplus_{\mu \in\left(\mathfrak{h}_{0}\right)^{*}} W^{\Gamma}(\lambda)_{\mu}
$$

and $W^{\Gamma}(\lambda)_{\mu} \neq 0$ only if $\mu \in \lambda-Q_{0}^{+}$. Further, we get $W^{\Gamma}(\lambda)_{\mu} \neq 0$ if and only if $W^{\Gamma}(\lambda)_{w(\mu)} \neq 0$ for all $w \in W_{0}$.

(2) As a $\mathfrak{g}_{0}$-module $W^{\Gamma}(\lambda)$ and $W^{\Gamma}(\lambda)[s]$ decompose into finite-dimensional irreducible representations of $\mathfrak{g}_{0}$.

(3) Let $\mu$ be a dominant integral weight such that $\lambda-\mu$ is as well a dominant integral. Then there exists a canonical homomorphism $W^{\Gamma}(\lambda) \rightarrow W^{\Gamma}(\mu) \otimes$ $W^{\Gamma}(\lambda-\mu)$ mapping $w_{\lambda}$ to $w_{\mu} \otimes w_{\lambda-\mu}$.

Proof. It suffices to show that for every $v \in W^{\Gamma}(\lambda)_{\mu}$ the module $U\left(\mathfrak{g}_{0}\right) . v$ is finitedimensional, since this proves the non-trivial statements in parts (1) and (2). Part (3) is clear from the defining relations. Given $v \in W^{\Gamma}(\lambda)_{\mu}$ we obtain $\mathbf{U}\left(\mathfrak{g}_{0}\right) \cdot v=$ $\mathbf{U}\left(\left(\mathfrak{n}_{-}\right)_{0}\right) \mathbf{U}\left(\mathfrak{n}_{0}\right) . v$. From part (1) we obtain that $U\left(\mathfrak{n}_{0}\right) \cdot v$ is finite-dimensional. By the PBW-theorem $U\left(\left(\mathfrak{n}_{-}\right)_{0}\right)$ is spanned by monomials, so it suffices to show that $X_{\beta, 0}^{-} \in\left(\mathfrak{n}_{-}\right)_{0}$ acts nilpotently on $v$. Assume that $v \in U(\mathfrak{C}(\widehat{\mathfrak{g}})) w_{\lambda}$ and the action of $\left(\mathfrak{n}_{-}\right)_{0}$ on $\mathfrak{C}(\widehat{\mathfrak{g}})$, which is given by the Lie bracket, is locally nilpotent. We obtain with

$$
\left(X_{\beta, 0}^{-} \otimes 1\right)^{\lambda\left(\beta^{\vee}\right)+1} w_{\lambda}=0, \quad\left(X_{\beta, 0}^{-}\right)^{N}(\underbrace{u . w_{\lambda}}_{=v})=\sum_{k=0}^{N}\left(\begin{array}{c}
N \\
k
\end{array}\right)\left(\left(X_{\beta, 0}^{-}\right)^{k} u\right)\left(X_{\beta, 0}^{-}\right)^{N-k} w_{\lambda}
$$

that $X_{\beta, 0}^{-}$acts nilpotently on $v$, which finally implies that $\mathbf{U}\left(\mathfrak{g}_{0}\right) \cdot v$ is finite-dimensional.

Remark 4.16. $W^{\Gamma}(\lambda)$ is finite-dimensional. This will be an immediate consequence of Theorem 5.1 and Corollary 7.4 .

By definition we obtain some obvious maps between Weyl modules and certain Demazure modules. 
Corollary 4.17. Let $\lambda$ be a dominant integral weight for $\mathfrak{g}_{0}$. Then for all $k \in$ $\left(1 / a_{0}^{\vee}\right) \mathbb{Z}_{>0}$ such that $(\lambda, k) \in X$, the Demazure module $D(k, \lambda)$ is a quotient of the Weyl module $W^{\Gamma}(\lambda)$.

Proof. This follows immediately by comparing the relations for the Weyl module in Definition 4.13 and the relations for the Demazure module in Corollary 4.9.

In this paper we want to show that the map between Weyl and Demazure modules is in fact an isomorphism. This is already known for untwisted current algebras of simply-laced type $([9],[6],[15])$. We recall the result for $\mathfrak{g}=\mathfrak{s l}_{2}$ here, since this will be heavily used throughout this paper.

Theorem 4.18. For $\mathfrak{g}=\mathfrak{s l}_{2}$ and $n \omega \in P^{+}$, we have an isomorphism of $\mathfrak{s l}_{2} \otimes \mathbb{C}[t]$ modules

$$
W(n \omega) \cong D(1, n \omega) .
$$

\section{Connection Between Weyl modules and Demazure modules}

In this section we will show that almost all Weyl modules are isomorphic to certain Demazure modules, e.g. the map in Corollary 4.17 is in fact an isomorphism.

Theorem 5.1. Suppose $\widehat{\mathfrak{g}}$ is of type $A_{2 l-1}^{(2)}, D_{l+1}^{(2)}, E_{6}^{(2)}$ or $D_{4}^{(3)}$. Then we have an isomorphism of $\mathfrak{C}(\widehat{\mathfrak{g}})$-modules

$$
W^{\Gamma}(\lambda) \cong D\left(1 / a_{0}^{\vee}, \lambda\right) .
$$

If $\widehat{\mathfrak{g}}$ is of type $A_{2 l}^{(2)}$ and $\lambda=\sum_{i=1}^{l} m_{i} \omega_{i}$ is a dominant weight such that $m_{l}$ is odd, we have an isomorphism of $\mathfrak{C}(\widehat{\mathfrak{g}})$-modules

$$
W^{\Gamma}(\lambda) \cong D\left(1 / a_{0}^{\vee}, \lambda\right) .
$$

Proof. By Corollary 4.17 we already know that the Demazure module is a quotient of the Weyl module. By comparing the defining relations in Corollary 4.9 and in Definition 4.13, we see that to prove that this map is an isomorphism, it is sufficient to show that the generator of the Weyl module is subject to the following relations:

For all $0 \leq j \leq m-1, \beta \in \Phi_{j}^{+}$,

$\left(X_{\beta, j}^{-} \otimes t^{m s+j}\right)^{k_{\beta}+1} \cdot w_{\lambda}=0$, where $s \geq 0, k_{\beta}=\max \left\{0,\left\langle\lambda, \beta^{\vee}\right\rangle-\frac{2(m s+j)}{\langle\beta, \beta\rangle} \frac{1}{a_{0}^{\vee}} a_{0}^{\vee}\right\}$.

Assume $\widehat{\mathfrak{g}}$ is not of type $A_{2 l}^{(2)}$. Then (5.1) is equivalent to

$$
\begin{aligned}
& \left(X_{\beta, j}^{-} \otimes t^{m s+j}\right)^{k_{\beta}+1} . w_{\lambda}=0, \\
& \text { where } s \geq 0, k_{\beta}=\left\{\begin{array}{cl}
\max \left\{0,\left\langle\lambda, \beta^{\vee}\right\rangle-s\right\}, & \text { if } \beta \text { is long, } \\
\max \left\{0,\left\langle\lambda, \beta^{\vee}\right\rangle-(m s+j)\right\}, & \text { if } \beta \text { is short. }
\end{array}\right.
\end{aligned}
$$

Let $\beta \in \Phi_{0}^{+}$be a long root and $V=U\left(\mathfrak{s l}_{2, \beta} \otimes \mathbb{C}\left[t^{m}\right]\right) . w_{\lambda} \subseteq W^{\Gamma}(\lambda)$ be the $\mathfrak{s l}_{2, \beta} \otimes$ $\mathbb{C}\left[t^{m}\right]$-submodule. Further, let $W\left(\left\langle\lambda, \beta^{\vee}\right\rangle \omega\right)$ be the $\mathfrak{s l}_{2} \otimes \mathbb{C}[t]$-Weyl module, which is by Theorem 4.18 isomorphic to the $\mathfrak{s l}_{2} \otimes \mathbb{C}[t]$-Demazure module $D\left(1,\left\langle\lambda, \beta^{\vee}\right\rangle \omega\right)$. Since $w_{\lambda}$ is a cyclic generator for $V$ and obviously satisfies the defining relations of $W\left(\left\langle\lambda, \beta^{\vee}\right\rangle \omega\right)$, we obtain by Lemma 3.1 a surjective homomorphism:

$$
W\left(\left\langle\lambda, \beta^{\vee}\right\rangle \omega\right) \cong D\left(1,\left\langle\lambda, \beta^{\vee}\right\rangle \omega\right) \rightarrow V \subseteq W^{\Gamma}(\lambda) .
$$


In particular, $w_{\lambda}$ satisfies the defining relations of $D\left(1,\left\langle\lambda, \beta^{\vee}\right\rangle \omega\right)$, which contain the relation

$$
\left(x^{-} \otimes t^{s}\right)^{\max \left\{0,\left\langle\lambda, \beta^{\vee}\right\rangle-s\right\}+1} \cdot v=0 \forall s \in \mathbb{N} .
$$

Therefore again by Lemma 3.1 we obtain

$$
\left(X_{\beta, 0}^{-} \otimes t^{m s}\right)^{\max \left\{0,\left\langle\lambda, \beta^{\vee}\right\rangle-s\right\}+1} \cdot w_{\lambda}=0 .
$$

Now suppose $\beta$ is a short root and consider the $\mathfrak{s l}_{2, \beta} \otimes \mathbb{C}[t]$-submodule $V=U\left(\mathfrak{s l}_{2, \beta} \otimes\right.$ $\mathbb{C}[t]) \cdot w_{\lambda} \subseteq W^{\Gamma}(\lambda)$. By the same reasons as above and Lemma 3.1 we get a surjective homomorphism

$$
W\left(\left\langle\lambda, \beta^{\vee}\right\rangle \omega\right) \cong D\left(1,\left\langle\lambda, \beta^{\vee}\right\rangle \omega\right) \rightarrow V \subseteq W^{\Gamma}(\lambda),
$$

and therefore $w_{\lambda}$ again satisfies the relations of $D\left(1,\left\langle\lambda, \beta^{\vee}\right\rangle \omega\right)$. Using the isomorphism in Lemma 3.1 we obtain

$$
\left(X_{\beta, j}^{-} \otimes t^{m s+j}\right)^{\max \left\{0,\left\langle\lambda, \beta^{\vee}\right\rangle-(m s+j)\right\}+1} \cdot w_{\lambda}=0, \forall s \in \mathbb{N}, 0 \leq j \leq m-1,
$$

which proves (5.2).

To prove the theorem it remains to consider the case where $\widehat{\mathfrak{g}}$ is of type $A_{2 l}^{(2)}$. We have $(\lambda, 1 / 2,0) \in X$, in particular, we have $D\left(1 / a_{0}^{\vee}, \lambda\right)=V_{\omega_{0} t_{\lambda-\omega_{l}}}\left(\Lambda_{l}\right)$. In order to again use Corollary 4.9 we reformulate (5.1) into

$$
\begin{aligned}
& \left(X_{\beta, j}^{-} \otimes t^{m s+j}\right)^{k_{\beta}+1} . w_{\lambda}=0, \\
& s \geq 0, k_{\beta}=\left\{\begin{array}{cl}
\max \left\{0,\left\langle\lambda, \beta^{\vee}\right\rangle-(m s+j)\right\}, & \text { if } \beta \text { is long, } \\
\max \left\{0,\left\langle\lambda, \beta^{\vee}\right\rangle-2(m s+j)\right\}, & \text { if } \beta \text { is short, } \\
\max \left\{0,\left\langle\lambda, \beta^{\vee}\right\rangle-1 / 2(m s+1)\right\}, & \text { if } \beta=2 \alpha, \alpha \text { is short. }
\end{array}\right.
\end{aligned}
$$

We will prove case by case that the generator of $W^{\Gamma}(\lambda)$ satisfies the relations in (5.3). For long roots the proof is similar to the other cases by using Lemma 3.2. So let $\beta$ be a short root and $\left\langle X_{\beta, j}^{ \pm} \otimes t^{m s+j}, X_{2 \beta, 1}^{ \pm} \otimes t^{m s+1}, h_{\beta, j} \otimes t^{m s+j}\right\rangle_{\mathbb{C}}$ the Lie algebra which is isomorphic to $\mathfrak{C}\left(A_{2}^{(2)}\right)$ by Lemma 3.2. We consider the submodule $U\left(\mathfrak{C}\left(A_{2}^{(2)}\right)\right) \cdot w_{\lambda} \subseteq W^{\Gamma}(\lambda)$, which is trivially a quotient of the $A_{2}^{(2)}$-Weyl module $W^{\Gamma}\left(\left\langle\lambda, \beta^{\vee}\right\rangle \omega\right)$. In Section 7, Theorem 7.1 we prove (independent of Sections 1-6) that $W^{\Gamma}\left(\left\langle\lambda, \beta^{\vee}\right\rangle \omega\right) \cong D\left(1 / 2,\left\langle\lambda, \beta^{\vee}\right\rangle \omega\right)$. The proof is finished with the observation that the defining relations for the $A_{2}^{(2)}$-Demazure module $D\left(1 / 2,\left\langle\lambda, \beta^{\vee}\right\rangle \omega\right)$ contain the relations

$$
\begin{gathered}
\left(X_{\beta, j}^{-} \otimes t^{m s+j}\right)^{\max \left\{0,\left\langle\lambda, \beta^{\vee}\right\rangle-2(m s+1)\right\}+1} \cdot w=0, \\
\left(X_{2 \beta, 1}^{-} \otimes t^{m s+1}\right)^{\max \left\{0,1 / 2\left(\left\langle\lambda, \beta^{\vee}\right\rangle-(m s+1)\right)\right\}+1} \cdot w=0 .
\end{gathered}
$$

5.1. Fundamental Weyl modules. In the previous section we have seen that Weyl modules are isomorphic to certain Demazure modules. Since most of the Demazure modules have a nice tensor product decomposition (see Theorem 4.11), we can transfer this result to most Weyl modules (only the $A_{2 l}^{(2)}$ case needs more work). Using this decomposition, to compute the dimension and character of Weyl modules it is enough to describe the $\mathfrak{g}_{0}$ decomposition of fundamental Weyl modules $W^{\Gamma}\left(\omega_{i}\right)$. 
Theorem 5.2. Let $\omega_{1}, \ldots, \omega_{l}$ be the fundamental weights in $P_{0}^{+}$. Viewed as a $\mathfrak{g}_{0^{-}}$ module, the fundamental Weyl module decomposes into the direct sum of irreducible $\mathfrak{g}_{0}$-modules as follows:

- if $\widehat{\mathfrak{g}}$ is of type $A_{2 l}^{(2)}$,

$$
\begin{aligned}
& W^{\Gamma}\left(\omega_{i}\right) \cong V\left(\omega_{i}\right), \\
& W^{\Gamma}\left(2 \omega_{l}\right) \cong V\left(2 \omega_{l}\right),
\end{aligned}
$$

- if $\widehat{\mathfrak{g}}$ is of type $A_{2 l-1}^{(2)}$,

$W^{\Gamma}\left(\omega_{i}\right) \cong \bigoplus_{s_{\bar{i}}+\cdots+s_{i}=1} V\left(s_{\bar{i}} \omega_{\bar{i}}+\cdots+s_{i-2} \omega_{i-2}+s_{i} \omega_{i}\right)$, where $\bar{i} \in\{0,1\}$ and $i=\bar{i} \bmod 2$,

- if $\widehat{\mathfrak{g}}$ is of type $D_{l+1}^{(2)}$,

$$
\begin{aligned}
& W^{\Gamma}\left(\omega_{i}\right) \cong \bigoplus_{s_{1}+\cdots+s_{i} \leq 1} V\left(s_{1} \omega_{1}+\cdots+s_{i} \omega_{i}\right), i \neq l, \\
& W^{\Gamma}\left(\omega_{l}\right) \cong V\left(\omega_{l}\right),
\end{aligned}
$$

- if $\widehat{\mathfrak{g}}$ is of type $E_{6}^{(2)}$,

$$
\begin{aligned}
& W^{\Gamma}\left(\omega_{1}\right) \cong \bigoplus_{s \leq 1} V\left(s \omega_{1}\right), \\
& W^{\Gamma}\left(\omega_{2}\right) \cong V(0) \oplus V\left(\omega_{1}\right)^{\oplus 2} \oplus V\left(\omega_{2}\right) \oplus V\left(\omega_{4}\right), \\
& W^{\Gamma}\left(\omega_{3}\right) \cong V(0)^{\oplus 2} \oplus V\left(\omega_{1}\right)^{\oplus 4} \oplus V\left(\omega_{2}\right)^{\oplus 3} \oplus V\left(\omega_{4}\right)^{\oplus 3} \oplus V\left(2 \omega_{1}\right) \oplus V\left(\omega_{1}+\omega_{4}\right) \oplus V\left(\omega_{3}\right), \\
& W^{\Gamma}\left(\omega_{4}\right) \cong \bigoplus_{s_{1}+s_{4} \leq 1} V\left(s_{1} \omega_{1}+s_{4} \omega_{4}\right),
\end{aligned}
$$

- if $\widehat{\mathfrak{g}}$ is of type $D_{4}^{(3)}$,

$$
\begin{aligned}
& W^{\Gamma}\left(\omega_{1}\right) \cong V(0) \oplus V\left(\omega_{1}\right) \oplus V\left(\omega_{2}\right)^{\oplus 2}, \\
& W^{\Gamma}\left(\omega_{2}\right) \cong \bigoplus_{s \leq 1} V\left(s \omega_{2}\right) .
\end{aligned}
$$

Proof. If $\widehat{\mathfrak{g}}$ is of type $A_{2 l-1}^{(2)}$ or $D_{l+1}^{(2)}$, the decomposition rule is immediate from Theorem 5.1 and Theorem 2 in [14]. By the same reasons the theorem is true for $i=2$ if $\widehat{\mathfrak{g}}$ is of type $D_{4}^{(3)}$ and for $i=1,4$ in type $E_{6}^{(2)}$. For $i=1$ one can check $t_{-w_{1}}=w_{0} s_{0} s_{2} s_{1} s_{2} s_{0}$, and therefore with the Demazure character formula we get

$$
\begin{aligned}
D_{t_{-w_{1}}}\left(e^{\Lambda_{0}}\right) & =D_{w_{0}}\left(e^{0}+2 e^{\omega_{2}}+e^{\omega_{1}}\right) \Rightarrow W^{\Gamma}\left(\omega_{1}\right) \cong V_{t_{-\omega_{1}}}\left(e^{\Lambda_{0}}\right) \\
& \cong G_{2} V(0) \oplus V\left(\omega_{1}\right) \oplus V\left(\omega_{2}\right)^{\oplus 2},
\end{aligned}
$$

which proves the claim for type $D_{4}^{(3)}$.

So it remains to consider the nodes $i=2,3$ in type $E_{6}^{(2)}$ and the general case in type $A_{2 l}^{(2)}$. In [7] Kirillov-Reshetikhin modules $K R\left(s \omega_{i}\right)$, respectively $K R^{\sigma}\left(s \omega_{i}\right)$, for the twisted version are defined. By inspecting the defining relations it follows that KR-modules of level 1 (e.g. $s=1$ ) are precisely fundamental Weyl modules, in particular

$$
W\left(\omega_{i}\right) \cong K R\left(\omega_{i}\right) \text { and } W^{\Gamma}\left(\omega_{i}\right) \cong K R^{\sigma}\left(\omega_{i}\right)
$$


Since the decomposition of KR-modules are known as $\mathfrak{g}$ - (respectively $\mathfrak{g}_{0^{-}}$)modules (see [20], 3], 18] or 7] for instance) we obtain the predicted decomposition for $i=2,3$ in type $E_{6}^{(2)}$ and for the general case in type $A_{2 l}^{(2)}$.

It remains to consider $W^{\Gamma}\left(2 \omega_{l}\right)$, so let $\left\langle X_{\alpha_{l}, j}^{ \pm} \otimes t^{m s+j}, X_{2 \alpha_{l}, 1}^{ \pm} \otimes t^{m s+1}, h_{\alpha_{l}, j} \otimes\right.$ $t^{m s+j}|s \in \mathbb{N}, 0 \leq j \leq m-1\rangle_{\mathbb{C}}$ be the Lie algebra which is by Lemma 3.2 isomorphic to $\mathfrak{C}\left(A_{2}^{(2)}\right)$. Then we obtain a surjective homomorphism

$$
W^{\Gamma}(2 \omega) \rightarrow U\left(\mathfrak{C}\left(A_{2}^{(2)}\right)\right) \cdot w_{2 \omega_{l}} \subseteq W^{\Gamma}\left(2 \omega_{l}\right) .
$$

In Section 7 we will show that the $A_{2}^{(2)}$-Weyl module $W^{\Gamma}(2 \omega)$ is an irreducible $\mathfrak{s l}_{2}$-module, and hence $\left(X_{\alpha_{l}, 0}^{-} \otimes t^{2}\right) \cdot w_{2 \omega_{l}}=\left(X_{2 \alpha_{l}, 1}^{-} \otimes t\right) \cdot w_{2 \omega_{l}}=\left(X_{\alpha_{l}, 1}^{-} \otimes t\right) \cdot w_{2 \omega_{l}}=0$. So $W^{\Gamma}\left(2 \omega_{l}\right)$ is isomorphic to the Kirillov-Reshetikhin module $K R^{\sigma}\left(2 \omega_{l}\right)$, hence the decomposition is known by [7].

Such a similar decomposition is already known for the untwisted fundamental Weyl modules $W\left(\omega_{i}\right)$; see [3] or [14] for instance. This fact motivates us to compare the dimension of twisted and untwisted fundamental Weyl modules. For notational reasons, we have to extend certain linear functions $\mathfrak{h}_{0} \longrightarrow \mathbb{C}$ to functions on $\mathfrak{h}$. So let $\mu \in P_{0}^{+}$(with $\mu\left(\alpha_{l}^{\vee}\right) \in 2 \mathbb{Z}_{\geq 0}$ if $\mathfrak{g}$ is of type $A_{2 l}$ ). We define the extension, by abuse of notation also denoted by $\mu$, on a basis of $\mathfrak{h}$, by

$$
\mu\left(h_{i}\right)=\left\{\begin{array}{cl}
\mu\left(\alpha_{i}^{\vee}\right) & \text { if } \mathfrak{g} \text { is not of type } A_{2 l}, \\
0 & \text { if } i \notin I_{0}, \\
\left(1-\frac{\delta_{i, l}}{2}\right) \mu\left(\alpha_{i}^{\vee}\right) & \text { if } \mathfrak{g} \text { is of type } A_{2 l} .
\end{array}\right.
$$

Since there might be confusion in the notation in the $A_{2 l}$ and the $l$-th fundamental weight case only, we will use this identification in the remainder of the paper without further comment.

Lemma 5.3. Let $\omega_{1}, \ldots, \omega_{l}$ be the fundamental weights in $P_{0}^{+}$. We set $\epsilon=\left(1+\delta_{i, l}\right)$ if $\mathfrak{g}$ is of type $A_{2 l}$ and $\epsilon=1$ otherwise. Then we obtain

$$
\operatorname{dim} W^{\Gamma}\left(\epsilon \omega_{i}\right)=\operatorname{dim} W\left(\epsilon \omega_{i}\right), 1 \leq i \leq l .
$$

Proof. Using Theorem 5.2, Theorem 2 in [14] and Lecture 24 in [17, we obtain the following straightforward calculations:

- if $\widehat{\mathfrak{g}}$ is of type $A_{2 l}^{(2)},\left(\mathfrak{g}, \mathfrak{g}_{0}\right)=\left(A_{2 l}, B_{l}\right)$ :

$$
\operatorname{dim} W^{\Gamma}\left(\epsilon \omega_{i}\right)=\left(\begin{array}{c}
2 l+1 \\
i
\end{array}\right)=\operatorname{dim}\left(V_{\mathfrak{g}}\left(\omega_{i}\right)\right)=\operatorname{dim} W\left(\epsilon \omega_{i}\right),
$$

- if $\widehat{\mathfrak{g}}$ is of type $A_{2 l-1}^{(2)},\left(\mathfrak{g}, \mathfrak{g}_{0}\right)=\left(A_{2 l-1}, C_{l}\right)$ :

$$
\operatorname{dim} W^{\Gamma}\left(\omega_{i}\right)=\left(\begin{array}{c}
2 l \\
\bar{i}
\end{array}\right)+\sum_{j=1}^{\frac{i-\bar{i}}{2}}\left(\begin{array}{c}
2 l \\
\bar{i}+2 j
\end{array}\right)-\left(\begin{array}{c}
2 l \\
\bar{i}+2 j-2
\end{array}\right)=\left(\begin{array}{c}
2 l \\
i
\end{array}\right)=\operatorname{dim} W\left(\omega_{i}\right),
$$

- if $\widehat{\mathfrak{g}}$ is of type $D_{l+1}^{(2)},\left(\mathfrak{g}, \mathfrak{g}_{0}\right)=\left(D_{l+1}, B_{l}\right)$ :

$$
\begin{aligned}
& \operatorname{dim} W^{\Gamma}\left(\omega_{i}\right)=\left\{\begin{array}{cl}
2^{i}, & \text { if } i=l, \\
1+\sum_{j=1}^{i}\left(\begin{array}{c}
2 l+1 \\
j
\end{array}\right), & i \neq l
\end{array}=\left\{\begin{array}{cl}
2^{i}, & \text { if } i=l, \\
\sum_{j=0}^{\frac{i-p_{i}}{2}}\left(\begin{array}{c}
2 l+2 \\
p_{i}+2 j
\end{array}\right), & i \neq l
\end{array}\right.\right. \\
& =\left\{\begin{array}{c}
\operatorname{dim} V_{\mathfrak{g}}\left(\omega_{l}\right), \text { if } i=l, \\
\operatorname{dim}\left(V_{\mathfrak{g}}\left(\omega_{i}\right) \oplus V_{\mathfrak{g}}\left(\omega_{i-2}\right) \oplus \cdots \oplus V_{\mathfrak{g}}\left(\omega_{p_{i}}\right)\right), i \neq l
\end{array}=\operatorname{dim} W\left(\omega_{i}\right),\right.
\end{aligned}
$$


- if $\widehat{\mathfrak{g}}$ is of type $E_{6}^{(2)},\left(\mathfrak{g}, \mathfrak{g}_{0}\right)=\left(E_{6}, F_{4}\right)$ :

$\operatorname{dim} W^{\Gamma}\left(\omega_{1}\right)=27=\operatorname{dim} V_{\mathfrak{g}}\left(\omega_{1}\right)=\operatorname{dim} W\left(\omega_{1}\right)$,

$\operatorname{dim} W^{\Gamma}\left(\omega_{2}\right)=378=\operatorname{dim}\left(\bigoplus_{s_{2}+s_{6}=1} V_{\mathfrak{g}}\left(s_{2} \omega_{2}+s_{6} \omega_{6}\right)\right)=\operatorname{dim} W\left(\omega_{2}\right)$,

$\operatorname{dim} W^{\Gamma}\left(\omega_{3}\right)=3732=\operatorname{dim}\left(V_{\mathfrak{g}}(0) \oplus V_{\mathfrak{g}}\left(\omega_{4}\right)^{\oplus 2} \oplus V_{\mathfrak{g}}\left(\omega_{1}+\omega_{6}\right) \oplus V_{\mathfrak{g}}\left(\omega_{3}\right)\right)=\operatorname{dim} W\left(\omega_{3}\right)$, $\operatorname{dim} W^{\Gamma}\left(\omega_{4}\right)=79=\operatorname{dim}\left(\bigoplus_{s_{4} \leq 1} V_{\mathfrak{g}}\left(s_{4} \omega_{4}\right)\right)=\operatorname{dim} W\left(\omega_{4}\right)$,

- if $\widehat{\mathfrak{g}}$ is of type $D_{4}^{(3)},\left(\mathfrak{g}, \mathfrak{g}_{0}\right)=\left(D_{4}, G_{2}\right)$ :

$$
\begin{aligned}
& \operatorname{dim} W^{\Gamma}\left(\omega_{1}\right)=29=\operatorname{dim}\left(V_{\mathfrak{g}}\left(\omega_{1}\right) \oplus V_{\mathfrak{g}}(0)\right)=\operatorname{dim} W\left(\omega_{1}\right), \\
& \operatorname{dim} W^{\Gamma}\left(\omega_{2}\right)=8=\operatorname{dim}\left(V_{\mathfrak{g}}\left(\omega_{2}\right)\right)=\operatorname{dim} W\left(\omega_{2}\right) .
\end{aligned}
$$

\section{Connection Between twisted And untwisted Weyl modules}

In this section we will show that the Weyl modules $W^{\Gamma}(\lambda)$ can be realized as associated graded modules of certain untwisted Weyl modules for the loop algebra $\mathfrak{g} \otimes \mathbb{C}\left[t, t^{-1}\right]$. So consider for $a \in \mathbb{C}^{*}$ the Lie algebra homomorphism $\varphi_{a}$ defined as follows:

$$
\varphi_{a}: \mathfrak{g} \otimes \mathbb{C}[t] \longrightarrow \mathfrak{g} \otimes \mathbb{C}[t], \quad x \otimes t^{m} \mapsto x \otimes(t+a)^{m} .
$$

For a $\mathfrak{g} \otimes \mathbb{C}[t]$-module $W$ we denote by $W_{a}$ the module obtained by pulling back $W$ through $\varphi_{a}$, i.e. $x \otimes t^{s}$ acts by $x \otimes(t+a)^{s}$. Further, we denote by $\bar{W}$ the module $W$ considered as a $\mathfrak{C}(\widehat{\mathfrak{g}})$-module, obtained by the embedding

$$
\mathfrak{C}(\widehat{\mathfrak{g}}) \hookrightarrow \mathfrak{g} \otimes \mathbb{C}[t] .
$$

For the definition of the associated graded modules in the following theorem, we refer to Section 3.1

Theorem 6.1. Let $\lambda=\sum_{i=1}^{l} m_{i} \omega_{i}$ be a dominant $\mathfrak{g}_{0}$-weight. If $\widehat{\mathfrak{g}}$ is a twisted Kac-Moody algebra not of type $A_{2 l}^{(2)}$, we get an isomorphism of $\mathfrak{C}(\widehat{\mathfrak{g}})$-modules:

$$
W^{\Gamma}(\lambda) \cong \operatorname{gr}\left(\overline{W_{a}(\lambda)}\right) .
$$

If $\widehat{\mathfrak{g}}$ is of type $A_{2 l}^{(2)}$ and $\lambda=\lambda_{1}+\lambda_{2} \in P_{0}^{+}$such that $m_{l}$ and $\lambda_{2}\left(\alpha_{l}^{\vee}\right)$ are odd, we get an isomorphism of $\mathfrak{C}(\widehat{\mathfrak{g}})$-modules:

$$
W^{\Gamma}(\lambda) \cong \operatorname{gr}\left(\overline{W_{a}\left(\lambda_{1}\right)} \otimes W^{\Gamma}\left(\lambda_{2}\right)\right) .
$$

Proof. Let $\widehat{\mathfrak{g}}$ not be of type $A_{2 l}^{(2)}$. By combining [13] and [5] it follows that $\overline{W_{a}(\lambda)}$ is a cyclic $\mathfrak{C}(\widehat{\mathfrak{g}})$-module. Therefore the associated graded is again cyclic, and it remains to observe that the image of the highest weight generator $\overline{\mathbf{w}}$ satisfies for $j \in\{0, \ldots, m-1\}$ and $h_{j} \in \mathfrak{h}_{j}$ the relations

$$
\begin{gathered}
\left(h_{j} \otimes t^{m s+j}\right) \cdot \overline{\mathbf{w}}=0, \quad(s, j) \neq(0,0), \\
\left(h_{0} \otimes 1\right) \overline{\mathbf{w}}=\lambda\left(h_{0}\right) \overline{\mathbf{w}} .
\end{gathered}
$$

Thus we obtain a surjective homomorphism

$$
W^{\Gamma}(\lambda) \rightarrow \operatorname{gr}\left(\overline{W_{a}(\lambda)}\right) .
$$


In order to compare the dimension of these modules we exploit the tensor product decomposition of $W^{\Gamma}(\lambda)$ as a $\mathfrak{g}_{0}$-module by combining Theorem 5.1 and Theorem 4.11. We obtain the following:

$$
W^{\Gamma}(\lambda) \cong W^{\Gamma}\left(\omega_{1}\right)^{\otimes m_{1}} \otimes \cdots \otimes W^{\Gamma}\left(\omega_{l}\right)^{\otimes m_{l}} \text { as } \mathfrak{g}_{0} \text {-modules. }
$$

An analogue decomposition was proven in [15] for untwisted Weyl modules for the current algebra of a simply-laced simple Lie algebra and is generalized in 25] for the non-simply-laced case. From this it follows immediately that

$$
\operatorname{dim} \operatorname{gr}\left(\overline{W_{a}(\lambda)}\right)=\operatorname{dim} W(\lambda)=\prod_{i=1}^{l}\left(\operatorname{dim} W\left(\omega_{i}\right)\right)^{m_{i}} .
$$

Hence by Lemma 5.3 we check that (6.1) is in fact an isomorphism.

From now on, we assume that $\widehat{\mathfrak{g}}$ is of type $A_{2 l}^{(2)}$. Since $\overline{W_{a}\left(\lambda_{1}\right)}$ and $W^{\Gamma}\left(\lambda_{2}\right)$ are cyclic $\mathfrak{C}(\widehat{\mathfrak{g}})$-modules, it follows with the usual arguments of [13] and the Chinese remainder theorem that the tensor product is cyclic as well. Therefore we obtain, similar to (6.1), a surjective homomorphism

$$
W^{\Gamma}(\lambda) \rightarrow \operatorname{gr}\left(\overline{W_{a}\left(\lambda_{1}\right)} \otimes W^{\Gamma}\left(\lambda_{2}\right)\right) .
$$

With the aim of comparing the dimension on both sides, we notice that

$$
\begin{aligned}
\operatorname{dim} \operatorname{gr}\left(\overline{W_{a}\left(\lambda_{1}\right)} \otimes W^{\Gamma}\left(\lambda_{2}\right)\right) & =\operatorname{dim} W\left(\lambda_{1}\right) \operatorname{dim} W^{\Gamma}\left(\lambda_{2}\right) \\
& =\prod_{i=1}^{l}\left(\operatorname{dim} W\left(\omega_{i}\right)\right)^{\lambda_{1}\left(\alpha_{i}^{\vee}\right)} \operatorname{dim} W^{\Gamma}\left(\lambda_{2}\right) .
\end{aligned}
$$

Our goal now is to prove the following tensor product decomposition:

$$
W^{\Gamma}(\lambda) \cong_{\mathfrak{g}_{0}} W^{\Gamma}\left(\omega_{1}\right)^{\otimes m_{1}} \otimes \cdots \otimes W^{\Gamma}\left(\omega_{l-1}\right)^{\otimes m_{l-1}} \otimes W^{\Gamma}\left(2 \omega_{l}\right)^{\otimes k-1} \otimes W^{\Gamma}\left(\omega_{l}\right),
$$

where $m_{l}=2 k-1$ since the proposition is an immediate consequence of (6.4) and Lemma 5.3. To prove (6.4) we investigate the character of $W^{\Gamma}(\lambda)$. By Theorem 5.1 and Theorem 4.6 we obtain

$$
\text { Char } W^{\Gamma}(\lambda)=\operatorname{Char} V_{\omega_{0} t_{\lambda-\omega_{l}}}\left(\Lambda_{l}\right)=D_{\omega_{0} t_{\lambda-\omega_{l}}}\left(e^{\Lambda_{l}}\right) .
$$

Suppose that $V(\mu)$ is an irreducible $B_{l}$-module such that the coefficient $n_{l}$ is even, whereby $\mu=\sum_{i=1}^{l} n_{i} \omega_{i}$. The first step will be to show that Char $V(\mu)$ is stable under the Demazure operators $D_{i}, i=0, \ldots, l$. The character of a finitedimensional $\mathfrak{g}_{0}$-module is stable under the Weyl group $W$ and hence stable under $D_{i}, i=1, \ldots, l$. It remains to consider the case $i=0$. Note that $\alpha_{0}=\delta-2 \bar{\theta}=\delta-\theta$, where $\bar{\theta}=\alpha_{1}+\cdots+\alpha_{l}$ is the highest short root of $B_{l}$. We define the maps $s_{\bar{\theta}}:\left(\mathfrak{h}_{0}\right)^{*} \rightarrow\left(\mathfrak{h}_{0}\right)^{*}, s_{\bar{\theta}}(\lambda)=\lambda-\lambda\left(\bar{\theta}^{\vee}\right) \bar{\theta}$ and $s_{\theta}:\left(\mathfrak{h}_{0}\right)^{*} \rightarrow\left(\mathfrak{h}_{0}\right)^{*}, s_{\theta}(\lambda)=\lambda-\lambda\left(\theta^{\vee}\right) \theta$. Since $\bar{\theta}^{\vee}=2\left(\alpha_{1}^{\vee}+\cdots+\alpha_{l-1}^{\vee}\right)+\alpha_{l}^{\vee}$ and $\theta^{\vee}=\alpha_{1}^{\vee}+\cdots+\alpha_{l-1}^{\vee}+\frac{1}{2} \alpha_{l}^{\vee}$, we clearly get $s_{\bar{\theta}}=s_{\theta}$. Thus $\nu$ is a weight in $V(\mu)$ if and only if $s_{\theta}(\nu)$ is a weight. Assume $\nu \in\left(\mathfrak{h}_{0}\right)^{*}$ is a weight, hence $\nu=\mu-Q_{0}^{+}$and therefore $\left\langle\nu, \alpha_{0}^{\vee}\right\rangle=\left\langle\nu,(\delta-\theta)^{\vee}\right\rangle=\left\langle\nu,-\theta^{\vee}\right\rangle \in \mathbb{Z}$. We have proved that $D_{0}$ can be defined on Char $V(\mu)$ and $D_{0}=D_{-\theta}$. We obtain

$$
D_{0}(\operatorname{Char} V(\mu))=D_{-\theta}(\operatorname{Char} V(\mu))=\operatorname{Char} V(\mu) .
$$


In a second step we prove that the characters are the same by using induction on $\sum_{i=1}^{l-1} m_{i}+(k-1)$. So if the sum is 1 we have to show

$$
\begin{gathered}
D_{\omega_{0} t_{\omega_{i}}}\left(e^{\Lambda_{l}}\right)=\text { Char } W^{\Gamma}\left(\omega_{i}+\omega_{l}\right)=e^{\frac{1}{2} \Lambda_{0}} \operatorname{Char}\left(V_{\mathfrak{g}_{0}}\left(\omega_{i}\right) \otimes V_{\mathfrak{g}_{0}}\left(\omega_{l}\right)\right), i<l, \\
D_{\omega_{0} t_{2 \omega_{l}}}\left(e^{\Lambda_{l}}\right)=\operatorname{Char} W^{\Gamma}\left(3 \omega_{l}\right)=e^{\frac{1}{2} \Lambda_{0}} \operatorname{Char}\left(V_{\mathfrak{g}_{0}}\left(2 \omega_{l}\right) \otimes V_{\mathfrak{g}_{0}}\left(\omega_{l}\right)\right) .
\end{gathered}
$$

In other words, we have to figure out the $\mathfrak{g}_{0}$-module decomposition of $W^{\Gamma}\left(\omega_{i}+\omega_{l}\right)$, respectively $W^{\Gamma}\left(3 \omega_{l}\right)$. By Lemma 4.15) (2) we already know that there exists such a decomposition, and since the modules are finite-dimensional every $\mathfrak{g}_{0}$-submodule is a direct summand. So our assignment is to find all highest weight vectors, first beginning with the highest weight vectors living in $W^{\Gamma}\left(\omega_{i}+\omega_{l}\right)[1]$. Suppose $\alpha \in \Phi_{1}$ such that $\left(X_{\alpha, 1}^{-} \otimes t\right) . w$ is a highest weight vector, i.e. the element is non-zero and the upper triangular part of $\mathfrak{g}_{0}$ acts by zero. We want to restrict the choice of $\alpha$ to one possible case. Note that $\alpha$ is of the form $\alpha_{j}+\cdots+\alpha_{l}$ or $2\left(\alpha_{j}+\cdots+\alpha_{l}\right)$, $1 \leq j \leq l$, or of the form $\alpha_{p}+\cdots+\alpha_{q}$, respectively $\alpha_{p}+\cdots+\alpha_{q-1}+2\left(\alpha_{q}+\cdots+\alpha_{l}\right)$, $p, q \leq l-1$. If $\alpha$ is a short root, we obtain from Lemma 3.2 that

$W^{\Gamma}\left(\left\langle\omega_{i}+\omega_{l}, \alpha^{\vee}\right\rangle \omega\right) \rightarrow U\left(\left\langle X_{\alpha, j}^{ \pm} \otimes t^{m s+j}, X_{2 \alpha, 1}^{ \pm} \otimes t^{m s+1}, h_{\alpha, j} \otimes t^{m s+j}\right\rangle_{\mathbb{C}} \cong \mathfrak{C}\left(A_{2}^{(2)}\right)\right) . w$,

whereby $W^{\Gamma}\left(\left\langle\omega_{i}+\omega_{l}, \alpha^{\vee}\right\rangle \omega\right)$ is the Weyl module for type $A_{2}^{(2)}$. So if $j>i$ in the representation of $\alpha$ as a sum of simple roots, we get $\left\langle\omega_{i}+\omega_{l}, \alpha^{\vee}\right\rangle=1$. In Section 7 it is shown that $W^{\Gamma}(\omega)$ is irreducible and therefore $\left(X_{\alpha, 1}^{-} \otimes t\right) \cdot w=\left(X_{2 \alpha, 1}^{-} \otimes t\right) \cdot w=0$. Now assume $j<i$ and $\left(X_{\alpha_{j}+\cdots+\alpha_{l}, 1}^{-} \otimes t\right) . w \neq 0$ is a highest weight vector. Hence $0=\left(X_{\alpha_{j}+\cdots+\alpha_{i-1}, 0}^{+} \otimes 1\right)\left(X_{\alpha_{j}+\cdots+\alpha_{l}, 1}^{-} \otimes t\right) . w=\left(X_{\alpha_{i}+\cdots+\alpha_{l}, 1}^{-} \otimes t\right) . w$, which is a contradiction to (6.3). In almost the same manner one sees that $\left(X_{2\left(\alpha_{j}+\cdots+\alpha_{l}\right), 1}^{-} \otimes\right.$ $t) . w$ can't be a highest weight vector. If $\alpha$ is a long root, we get with Lemma 3.2 that

$$
W\left(\left\langle\omega_{i}+\omega_{l}, \alpha^{\vee}\right\rangle \omega\right) \rightarrow U\left(\mathfrak{s l}_{2, \alpha} \otimes \mathbb{C}[t]\right) . w,
$$

whereby $W^{\Gamma}\left(\left\langle\omega_{i}+\omega_{l}, \alpha^{\vee}\right\rangle \omega\right)$ is the Weyl module for the current algebra $\mathfrak{s l}_{2} \otimes \mathbb{C}[t]$. So if $\alpha=\alpha_{p}+\cdots+\alpha_{q}$, we again obtain $\left\langle\omega_{i}+\omega_{l}, \alpha^{\vee}\right\rangle \leq 1$ and therefore $\left(X_{\alpha, 1}^{-} \otimes t\right) . w=0$. Let $\alpha$ be of the form $\alpha_{p}+\cdots+\alpha_{q-1}+2\left(\alpha_{q}+\cdots+\alpha_{l}\right)$ such that $i \geq p$ and $\left(X_{\alpha, 1}^{-} \otimes t\right) . w$ is a non-zero highest weight vector. Therefore the upper triangular part acts by zero, especially

$$
\begin{aligned}
0 & =\left(X_{\alpha_{q}+\cdots+\alpha_{l}, 0}^{+} \otimes 1\right)\left(X_{\alpha_{p}+\cdots+\alpha_{i-1}, 0}^{+} \otimes 1\right)\left(X_{\alpha_{p}+\cdots+\alpha_{q-1}+2\left(\alpha_{q}+\cdots+\alpha_{l}\right), 1}^{-} \otimes t\right) \cdot w \\
& =\left(X_{\alpha_{q}+\cdots+\alpha_{l}, 0}^{+} \otimes 1\right)\left(X_{\alpha_{i}+\cdots+\alpha_{q-1}+2\left(\alpha_{q}+\cdots+\alpha_{l}\right), 1}^{-} \otimes t\right) \cdot w=\left(X_{\alpha_{i}+\cdots+\alpha_{l}, 1}^{-} \otimes t\right) . w,
\end{aligned}
$$

which is again a contradiction to 6.3 . Hence the only possibility to get a highest weight vector of degree one is to apply $\left(X_{\alpha_{i}+\cdots+\alpha_{l}, 1}^{-} \otimes t\right)$ on $w$. Clearly we have by Section $7\left(X_{\alpha_{i}+\cdots+\alpha_{l}, 1}^{-} \otimes t\right)^{2} \cdot w=\left(X_{\alpha_{i}+\cdots+\alpha_{l}, 1}^{-} \otimes t^{2 s+1}\right) \cdot w=\left(X_{\alpha_{i}+\cdots+\alpha_{l}, 0}^{-} \otimes t^{2 s}\right) \cdot w=0$ for $s \geq 1$, because in (6.5) we have $\left\langle\omega_{i}+\omega_{l},\left(\alpha_{i}+\cdots+\alpha_{l}\right)^{\vee}\right\rangle=3$. Thus one can check that $\left(X_{\alpha_{i}+\cdots+\alpha_{l}, 1}^{-} \otimes t\right) . w$ satisfies the relations (4.4), (4.5) in Definition 4.13 and has weight $\omega_{i-1}+\omega_{l}$ with respect to $\mathfrak{h}_{0}$. Hence the calculations above show on the one hand that $\left(X_{\alpha_{i}+\cdots+\alpha_{l}, 1}^{-} \otimes t\right) . w$ is really a highest weight vector, but on the other hand we get more than this, namely a surjective map

$$
W^{\Gamma}\left(\omega_{i-1}+\omega_{l}\right) \rightarrow U\left(\mathfrak{C}\left(A_{2 l}^{(2)}\right)\right)\left(X_{\alpha_{i}+\cdots+\alpha_{l}, 1}^{-} \otimes t\right) . w .
$$


Since $W^{\Gamma}\left(\omega_{i}+\omega_{l}\right)[1] \cong \mathfrak{g}_{\mathfrak{g}_{0}} V_{\mathfrak{g}_{0}}\left(\omega_{i-1}+\omega_{l}\right) \cong U\left(\mathfrak{g}_{0}\right)\left(X_{\alpha_{i}+\cdots+\alpha_{l}, 1}^{-} \otimes t\right) . w$ we obtain $W^{\Gamma}\left(\omega_{i}+\omega_{l}\right)=U\left(\mathfrak{g}_{0}\right) \cdot w \oplus U\left(\mathfrak{C}\left(A_{2 l}^{(2)}\right)\right)\left(X_{\alpha_{i}+\cdots+\alpha_{l}, 1}^{-} \otimes t\right) \cdot w \cong \cong_{\mathfrak{g}_{0}} V_{\mathfrak{g}_{0}}\left(\omega_{i}+\omega_{l}\right) \oplus$ $W^{\Gamma}\left(\omega_{i-1}+\omega_{l}\right) / I$, for some ideal $I$. Using (6.3) one can check that the ideal is zero, and therefore by induction we prove our claim, because for $i=1$ we get

$$
\begin{aligned}
\operatorname{Char}\left(V_{\omega_{0} t_{\omega_{1}}}\left(\Lambda_{l}\right)\right) & \cong V_{\omega_{0} s_{0} s_{1} \ldots s_{l}}\left(\Lambda_{l}\right)=D_{\omega_{0}} D_{0} \ldots D_{l}\left(e^{\Lambda_{l}}\right) \\
& =D_{\omega_{0}}\left(e^{\Lambda_{l}}+e^{\Lambda_{l}-\alpha_{l}}+\cdots+e^{\Lambda_{l}-\alpha_{l}-\cdots-\alpha_{1}}+e^{\Lambda_{l}+\omega_{1}}\right) \\
& =e^{\frac{1}{2} \Lambda_{0}} \operatorname{Char}\left(V_{\mathfrak{g}_{0}}\left(\omega_{1}+\omega_{l}\right) \oplus V_{\mathfrak{g}_{0}}\left(\omega_{l}\right)\right) \\
& =e^{\frac{1}{2} \Lambda_{0}} \operatorname{Char}\left(V_{\mathfrak{g}_{0}}\left(\omega_{1}\right) \otimes V_{\mathfrak{g}_{0}}\left(\omega_{l}\right)\right) .
\end{aligned}
$$

In exactly the same way one can prove the existence of a surjective map:

$$
W^{\Gamma}\left(\omega_{l-1}+\omega_{l}\right) \rightarrow U\left(\mathfrak{C}\left(A_{2 l}^{(2)}\right)\right)\left(X_{\alpha_{l}, 1}^{-} \otimes t\right) . w .
$$

Furthermore, a more simple calculation shows $W^{\Gamma}\left(3 \omega_{l}\right)[1] \cong \mathfrak{g}_{0} V_{\mathfrak{g}_{0}}\left(\omega_{l-1}+\omega_{l}\right) \cong$ $U\left(\mathfrak{g}_{0}\right)\left(X_{\alpha_{l}, 1}^{-} \otimes t\right) . w$. Hence $W^{\Gamma}\left(3 \omega_{l}\right) \cong_{\mathfrak{g}_{0}} V_{\mathfrak{g}_{0}}\left(2 \omega_{l}\right) \otimes V_{\mathfrak{g}_{0}}\left(\omega_{l}\right)$, which finally proves the initial step. So let $\sum_{i=1}^{l-1} m_{i}+(k-1)>1$ and $m_{i}, i<l$ or $k-1$, such that one of them is larger than or equal to 1 . Using Proposition 4.7 in the first case we get

$$
\begin{aligned}
& D_{\omega_{0} t_{\lambda-\omega_{l}}}\left(e^{\Lambda_{l}}\right)=D_{t_{-\omega_{i}}} D_{\omega_{0} t_{\lambda-\omega_{l}-\omega_{i}}}\left(e^{\Lambda_{l}}\right) \\
& =D_{t_{-\omega_{i}}}\left(e ^ { \frac { 1 } { 2 } \Lambda _ { 0 } } \operatorname { C h a r } \left(V_{\mathfrak{g}_{0}}\left(\omega_{1}\right)^{\otimes m_{1}} \otimes \cdots \otimes V_{\mathfrak{g}_{0}}\left(\omega_{i}\right)^{\otimes m_{i}-1}\right.\right. \\
& \left.\left.\otimes \cdots \otimes V_{\mathfrak{g}_{0}}\left(2 \omega_{l}\right)^{k-1} \otimes V_{\mathfrak{g}_{0}}\left(\omega_{l}\right)\right)\right) \\
& =\operatorname{Char}\left(V_{\mathfrak{g}_{0}}\left(\omega_{1}\right)^{\otimes m_{1}} \otimes \cdots \otimes V_{\mathfrak{g}_{0}}\left(\omega_{i}\right)^{\otimes m_{i}-1}\right. \\
& \left.\otimes \cdots \otimes V_{\mathfrak{g}_{0}}\left(2 \omega_{l}\right)^{k-1}\right) D_{t_{-\omega_{i}}}\left(e^{\frac{1}{2} \Lambda_{0}} \operatorname{Char}\left(V_{\mathfrak{g}_{0}}\left(\omega_{l}\right)\right)\right) \\
& =e^{\frac{1}{2} \Lambda_{0}} \operatorname{Char}\left(V_{\mathfrak{g}_{0}}\left(\omega_{1}\right)^{\otimes m_{1}} \otimes \cdots \otimes V_{\mathfrak{g}_{0}}\left(\omega_{i}\right)^{\otimes m_{i}} \otimes \cdots \otimes V_{\mathfrak{g}_{0}}\left(2 \omega_{l}\right)^{k-1} \otimes V_{\mathfrak{g}_{0}}\left(\omega_{l}\right)\right) .
\end{aligned}
$$

In the second we obtain

$$
\begin{aligned}
& D_{\omega_{0} t_{2(k-1) \omega_{l}}}\left(e^{\Lambda_{l}}\right)=D_{-t_{2 \omega_{l}}} D_{\omega_{0} t_{2(k-2) \omega_{l}}}\left(e^{\Lambda_{l}}\right) \\
& =D_{-t_{2 \omega_{l}}}\left(e^{\frac{1}{2} \Lambda_{0}} \operatorname{Char}\left(V_{\mathfrak{g}_{0}}\left(2 \omega_{l}\right)^{\otimes k-2} \otimes V_{\mathfrak{g}_{0}}\left(\omega_{l}\right)\right)\right) \\
& =\operatorname{Char}\left(V_{\mathfrak{g}_{0}}\left(2 \omega_{l}\right)^{\otimes k-2}\right) D_{-t_{2 \omega_{l}}}\left(e^{\frac{1}{2} \Lambda_{0}} \operatorname{Char}\left(V_{\mathfrak{g}_{0}}\left(\omega_{l}\right)\right)\right) \\
& =e^{\frac{1}{2} \Lambda_{0}} \operatorname{Char}\left(V_{\mathfrak{g}_{0}}\left(2 \omega_{l}\right)^{\otimes k-1} \otimes V_{\mathfrak{g}_{0}}\left(\omega_{l}\right)\right) .
\end{aligned}
$$

As an immediate consequence of Theorem 6.1 and its proof we obtain explicit dimension formulas for Weyl modules. Such formulas for Weyl modules, as already mentioned, were previously known for untwisted current algebras (see [6], [15] or [25]).

Corollary 6.2. Let $\lambda=\sum_{i=1}^{l} m_{i} \omega_{i}$ be a decomposition of a dominant weight $\lambda \in$ $P_{0}^{+}$.

(1) If $\widehat{\mathfrak{g}}$ is a twisted affine Kac-Moody algebra not of type $A_{2 l}^{(2)}(l \geq 1)$, then

$$
\operatorname{dim} W^{\Gamma}(\lambda)=\prod_{i=1}^{l}\left(\operatorname{dim} W^{\Gamma}\left(\omega_{i}\right)\right)^{m_{i}}=\prod_{i=1}^{l}\left(\operatorname{dim} W\left(\omega_{i}\right)\right)^{m_{i}} .
$$


(2) If $\widehat{\mathfrak{g}}$ is of type $A_{2 l}^{(2)}$ and $m_{l}=2 k-1$, then

$$
\begin{aligned}
\operatorname{dim} W^{\Gamma}(\lambda) & =\prod_{i=1}^{l-1}\left(\operatorname{dim} W^{\Gamma}\left(\omega_{i}\right)\right)^{m_{i}}\left(\operatorname{dim} W^{\Gamma}\left(2 \omega_{l}\right)\right)^{k-1} \operatorname{dim} W^{\Gamma}\left(\omega_{l}\right) \\
& =\left(\prod_{i=1}^{l-1}\left(\begin{array}{c}
2 l+1 \\
i
\end{array}\right)^{m_{i}}\right)\left(\begin{array}{c}
2 l+1 \\
l
\end{array}\right)^{k-1} 2^{l} .
\end{aligned}
$$

6.1. Constructions from arbitrary local Weyl modules. In the previous section we investigated the connection between untwisted and twisted Weyl modules. We have seen that the twisted ones can be realized as associated graded modules of certain untwisted Weyl modules located in a single point. In this section we generalize this result using untwisted Weyl modules located in a finite number of points.

Let $W^{1}, \ldots, W^{k}$ be finite-dimensional, graded and cyclic modules with cyclic vectors $w_{1}, \ldots, w_{k}$ for the current algebra, and further let $W$ be a given cyclic graded $\mathfrak{C}(\widehat{\mathfrak{g}})$-module (possibly trivial) with cyclic vector $w$.

Proposition 6.3. Let $a_{i} \in \mathbb{C}^{*}, 1 \leq i \leq k$, be non-zero complex numbers such that $a_{i}^{m} \neq a_{j}^{m}$ for $i \neq j$. Then $\overline{W_{a_{1}}^{1}} \otimes \cdots \otimes \overline{W_{a_{k}}^{k}} \otimes W$ is a cyclic $U(\mathfrak{C}(\widehat{\mathfrak{g}}))$-module, in particular we get

$$
\overline{W_{a_{1}}^{1}} \otimes \cdots \otimes \overline{W_{a_{k}}^{k}} \otimes W=U(\mathfrak{C}(\widehat{\mathfrak{g}})) \cdot(\mathbf{w} \otimes w) .
$$

Proof. As $W^{i}$ are finite-dimensional and graded, there exists a sufficiently large $N_{i}$ such that $x \otimes t^{s}$ acts trivially for $s \geq N_{i}$. Thus the ideal $J_{i}:=\mathfrak{g} \otimes\left(t-a_{i}\right)^{N_{i}} \mathbb{C}[t]$ acts trivially on $W_{a_{i}}^{i}$. We define $\eta: \mathbb{C}^{*} \rightarrow \mathbb{N}, a_{i} \mapsto N_{i}$. Then Supp $\eta$ do not contain two points in the same $\Gamma$-orbit, and therefore, similar to the proof of Theorem 6.1, we obtain that $\overline{W_{a_{1}}^{1}} \otimes \cdots \otimes \overline{W_{a_{k}}^{k}}$ is a cyclic $U(\mathfrak{C}(\widehat{\mathfrak{g}}))$-module. The rest is an application of the Chinese remainder theorem.

Remark 6.4. We can consider arbitrary g-modules $V\left(\lambda_{i}\right), \lambda_{i} \in P^{+}, 1 \leq i \leq k$, as graded and cyclic $\mathfrak{g} \otimes \mathbb{C}[t]$-modules, where the action is given by

$$
x \otimes f(t) . v=f(0) x . v, x \in \mathfrak{g}, f \in \mathbb{C}[t] .
$$

Hence if $W^{i}=V\left(\lambda_{i}\right)$, it is already shown in 22 or in a more general setting of equivariant map algebras in 26 that the tensor product in Proposition 6.3 is irreducible. Moreover, it is known that all finite-dimensional irreducible modules are tensor products of evaluation modules.

In [13] local Weyl modules for equivariant map algebras were defined and a tensor product property was proven. It was shown that if $W^{i}$ is an untwisted graded Weyl module, then $\overline{W_{a_{i}}^{i}}$ is a local Weyl module for $\mathfrak{C}(\widehat{\mathfrak{g}})$ supported in the point $a_{i}$. The tensor product property gives that $\overline{W_{a_{1}}\left(\lambda_{1}\right)} \otimes \cdots \otimes \overline{W_{a_{r}}\left(\lambda_{r}\right)}$ is a local Weyl module for $\mathfrak{C}(\widehat{\mathfrak{g}})$. It was shown that every local Weyl module of $\mathfrak{C}(\widehat{\mathfrak{g}})$ can be obtained in this way. The following corollary (in $A_{2 l}^{(2)}$ again only the odd-case is considered) shows that the dimension and $\mathfrak{g}_{0}$ character is independent of the support of the local Weyl module.

Corollary 6.5. Let $\lambda=\lambda_{1}+\cdots+\lambda_{r}$ be a decomposition of a dominant weight $\lambda \in P_{0}^{+}$into dominant weights and let $a_{1}, \ldots, a_{r} \in \mathbb{C}^{*}$ s.t. $a_{i}^{m} \neq a_{j}^{m}$ for $i \neq j$. 
(1) If $\widehat{\mathfrak{g}}$ is a twisted affine Kac-Moody algebra not of type $A_{2 l}^{(2)}$, then we have an isomorphism of $\mathfrak{C}(\widehat{\mathfrak{g}})$-modules:

$$
W^{\Gamma}(\lambda) \cong \operatorname{gr}\left(\overline{W_{a_{1}}\left(\lambda_{1}\right)} \otimes \cdots \otimes \overline{W_{a_{r}}\left(\lambda_{r}\right)}\right) .
$$

(2) If $\widehat{\mathfrak{g}}$ is of type $A_{2 l}^{(2)}$ and $\lambda_{i}\left(\alpha_{l}^{\vee}\right) \in 2 \mathbb{Z}_{\geq_{0}}$ for $1 \leq i \leq r-1$ and $\lambda_{r}\left(\alpha_{l}^{\vee}\right)$ is odd, then we get an isomorphism of $\mathfrak{C}(\widehat{\mathfrak{g}})$-modules:

$$
W^{\Gamma}(\lambda) \cong \operatorname{gr}\left(\overline{W_{a_{1}}\left(\lambda_{1}\right)} \otimes \cdots \otimes \overline{W_{a_{r-1}}\left(\lambda_{r-1}\right)} \otimes W^{\Gamma}\left(\lambda_{r}\right)\right) .
$$

Proof. By Proposition 6.3 the right hand side in (1), respectively (2), is cyclic. Hence it is easy to obtain a surjecive map of $\mathfrak{C}(\widehat{\mathfrak{g}})$-modules, which is by Theorem 6.1 clearly an isomorphism.

Remark 6.6. As mentioned in the introduction, Weyl modules are defined in 13 ] in a more general way, with support in $\mathbb{C}$. They are also parametrized by finitely supported functions from $\mathbb{C}$ to $P^{+}$. With this corollary we have shown in all cases, except the even case in $A_{2 l}^{(2)}$, that the dimension and $\mathfrak{g}_{0}$ character of a local Weyl module depends only on its $\mathfrak{g}_{0}$ maximal weight and NOT on the support of its parametrizing function. Concluding, one might be able to show that the global Weyl module is a free module for a certain algebra, which might be part of a forthcoming publication.

Remark 6.7. The same construction of an associated graded module out of finitedimensional, graded and cyclic $\mathfrak{g} \otimes \mathbb{C}[t]$-modules is defined in [11] and is called the fusion product. In the twisted case the same construction fails, since, for this, one would need a pullback map like

$$
\sum_{j=0}^{m-1}\left(x_{j} \otimes t^{m s+j}\right) \in \mathfrak{C}(\widehat{\mathfrak{g}}) \mapsto \sum_{j=0}^{m-1}\left(x_{j} \otimes(t+a)^{m s+j}\right) \notin \mathfrak{C}(\widehat{\mathfrak{g}}) .
$$

Therefore we have constructed in our results associated graded $\mathfrak{C}(\widehat{\mathfrak{g}})$-modules out of modules coming from $\mathfrak{g} \otimes \mathbb{C}[t]$, which represent an analogue of fusion products.

6.2. Summary of the results. As a conclusion we summarize our results: Let $\lambda=m_{1} \omega_{1}+\cdots+m_{l} \omega_{l}$ be a dominant weight of $\mathfrak{g}_{0}$ and $\epsilon=0$ if $l$ is odd and $\epsilon=1$ otherwise. Then

- if $\widehat{\mathfrak{g}}$ is of type $A_{2}^{(2)}(n$ is odd $)$,

$$
W^{\Gamma}(n \omega) \cong \operatorname{gr}\left({\overline{W\left(\omega_{1}\right)}}^{\otimes(k-1)} \otimes W^{\Gamma}(\omega)\right) \cong V_{s_{1} t_{(n-1) \omega}}\left(\Lambda_{1}\right),
$$

- if $\widehat{\mathfrak{g}}$ is of type $A_{2 l}^{(2)}\left(m_{l}\right.$ is odd),

$$
\begin{aligned}
W^{\Gamma}(\lambda) & \cong \operatorname{gr}\left(\overline{W\left(\omega_{1}\right)}{ }^{\otimes m_{1}} \otimes \cdots \otimes{\overline{W\left(\omega_{l-1}\right)}}^{\otimes m_{l-1}} \otimes{\overline{W\left(\omega_{l}\right)}}^{\otimes(k-1)} \otimes W^{\Gamma}\left(\omega_{l}\right)\right) \\
& \cong V_{\omega_{0} t_{\lambda-\omega_{l}}}\left(\Lambda_{l}\right),
\end{aligned}
$$

- if $\widehat{\mathfrak{g}}$ is of type $A_{2 l-1}^{(2)}$,

$$
\begin{aligned}
W^{\Gamma}(\lambda) & \cong \operatorname{gr}\left({\overline{W\left(\omega_{1}\right)}}^{\otimes m_{1}} \otimes \cdots \otimes{\overline{W\left(\omega_{l}\right)}}^{\otimes m_{l}}\right) \\
& \cong\left\{\begin{array}{cl}
V_{\omega_{0} t_{\lambda}}\left(\Lambda_{0}\right), & \text { if } m_{1}+3 m_{3}+\cdots+(l-\epsilon) m_{l-\epsilon} \text { is even }, \\
V_{\omega_{0} t_{\lambda-\omega_{1}}}\left(\Lambda_{1}\right), & \text { else, }
\end{array}\right.
\end{aligned}
$$


- if $\widehat{\mathfrak{g}}$ is of type $D_{l+1}^{(2)}$,

$W^{\Gamma}(\lambda) \cong \operatorname{gr}\left(\overline{W\left(\omega_{1}\right)}{ }^{\otimes m_{1}} \otimes \cdots \otimes \overline{W\left(\omega_{l}\right)}{ }^{\otimes m_{l}}\right) \cong\left\{\begin{array}{cl}V_{\omega_{0} t_{\lambda}}\left(\Lambda_{0}\right), & \text { if } m_{l} \text { is even, } \\ V_{\omega_{0} t_{\lambda-\omega_{l}}}\left(\Lambda_{l}\right), & \text { else, }\end{array}\right.$

- if $\widehat{\mathfrak{g}}$ is of type $E_{6}^{(2)}$,

$$
W^{\Gamma}(\lambda) \cong \operatorname{gr}\left({\overline{W\left(\omega_{1}\right)}}^{\otimes m_{1}} \otimes \cdots \otimes{\overline{W\left(\omega_{l}\right)}}^{\otimes m_{l}}\right) \cong V_{\omega_{0} t_{\lambda}}\left(\Lambda_{0}\right),
$$

- if $\widehat{\mathfrak{g}}$ is of type $D_{4}^{(3)}$,

$$
W^{\Gamma}(\lambda) \cong \operatorname{gr}\left({\overline{W\left(\omega_{1}\right)}}^{\otimes m_{1}} \otimes \cdots \otimes{\overline{W\left(\omega_{l}\right)}}^{\otimes m_{l}}\right) \cong V_{\omega_{0} t_{\lambda}}\left(\Lambda_{0}\right) .
$$

\section{Proofs For the type $A_{2}^{(2)}$ BASIC TWisted CASE}

In this section our attention is devoted to the twisted Kac-Moody algebra $A_{2}^{(2)}$. In the previous sections we claimed that the results already hold for $A_{2}^{(2)}$, so to complete our work we need to verify the following main result of this section.

Theorem 7.1. Let $n$ be an odd integer. Then the Weyl module $W^{\Gamma}(n \omega)$ is isomorphic to the Demazure module $D(1 / 2, n \omega) \cong V_{s_{1} t_{(n-1) \omega}}\left(\Lambda_{1}\right)$.

\subsection{Properties of $W^{\Gamma}(n \omega)$ Weyl modules and minimal powers.}

Lemma 7.2. Let $I^{\sigma}$ be the left ideal in $U\left(\mathfrak{C}\left(A_{2}^{(2)}\right)\right)$ generated by $\mathfrak{n}_{j} \otimes t^{j} C\left[t^{m}\right],\left(h_{\alpha, 0} \otimes\right.$ $\left.t^{2 r}\right),\left(h_{\alpha, 1} \otimes t^{2 r-1}\right), r \geq 1,0 \leq j \leq m-1$. Then for every $k \in \mathbb{N}_{+}$there exists a non-zero scalar $c_{k}, \tilde{c_{k}} \in \mathbb{C}$ such that

(1)

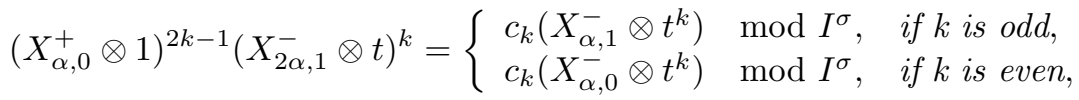

$$
\left(X_{2 \alpha, 1}^{+} \otimes t\right)^{k-1}\left(X_{2 \alpha, 1}^{-} \otimes t\right)^{k}=\tilde{c_{k}}\left(X_{2 \alpha, 1}^{-} \otimes t^{2 k-1}\right) \bmod I^{\sigma} .
$$

Proof. The first equation is a simple reformulation of Lemma 3.3 (iii) in [5]. We will prove the second equation by induction. For $k=1$ we trivially get $\tilde{c_{k}}=1$. Suppose that (2) is already true for all $p \leq k$. Then

$$
\begin{aligned}
\left(X_{2 \alpha, 1}^{+} \otimes t\right)^{k}\left(X_{2 \alpha, 1}^{-} \otimes t\right)^{k+1}=\left(X_{2 \alpha, 1}^{+} \otimes t\right)\left(X_{2 \alpha, 1}^{+} \otimes t\right)^{k-1}\left(X_{2 \alpha, 1}^{-} \otimes t\right)^{k}\left(X_{2 \alpha, 1}^{-} \otimes t\right) \\
=\tilde{c_{k}}\left(X_{2 \alpha, 1}^{+} \otimes t\right)\left(X_{2 \alpha, 1}^{-} \otimes t^{2 k-1}\right)\left(X_{2 \alpha, 1}^{-} \otimes t\right) \\
\quad+\left(X_{2 \alpha, 1}^{+} \otimes t\right) \mathfrak{J}\left(X_{2 \alpha, 1}^{-} \otimes t\right), \text { for some } \mathfrak{J} \in I^{\sigma} \\
\equiv-\frac{1}{2} \tilde{c_{k}}\left(h_{\alpha, 0} \otimes t^{2 k}\right)\left(X_{2 \alpha, 1}^{-} \otimes t\right) \quad \bmod I^{\sigma} \\
\equiv 2 \tilde{c_{k}}\left(X_{2 \alpha, 1}^{-} \otimes t^{2 k+1}\right) \quad \bmod I^{\sigma} .
\end{aligned}
$$

Corollary 7.3. Let $n \in \mathbb{N}$ such that $n=2 k$ if $n$ is even and $n=2 k-1$ if $n$ is odd. Then we have

(1) $\left(X_{2 \alpha, 1}^{-} \otimes t\right)^{k} w_{n}=0$, 
(2) $\begin{cases}\left(X_{\alpha, 0}^{-} \otimes t^{k}\right) w_{n}=\left(X_{\alpha, 1}^{-} \otimes t^{k+1}\right) w_{n}=0, & \text { if } k \text { is even, } \\ \left(X_{\alpha, 0}^{-} \otimes t^{k+1}\right) w_{n}=\left(X_{\alpha, 1}^{-} \otimes t^{k}\right) w_{n}=0, & \text { if } k \text { is odd, }\end{cases}$

(3) $\left(X_{2 \alpha, 1}^{-} \otimes t^{2 k-1}\right) w_{n}=0$.

Proof. Clearly parts (2) and (3) are deductions of Lemma 7.2 and part (1). Assume now that $\left(X_{2 \alpha, 1}^{-} \otimes t\right)^{k} w_{n}$ is a non-zero element in $W^{\Gamma}(n \omega)[k]$ of weight $-2 k \omega$ if $n$ is even and $(-2 k-1) \omega$ if $n$ is odd. Also recall that $W^{\Gamma}(n \omega)[k]$ is an integrable $\mathfrak{s l}_{2}$-module, i.e. Proposition 4.15 is applicable. That means $W^{\Gamma}(n \omega)[k]_{2 k \omega} \neq 0$, respectively $W^{\Gamma}(n \omega)[k]_{(2 k+1) \omega} \neq 0$, but both are impossible, which proves part (1).

Corollary 7.4. For all $n \in \mathbb{N}$ the modules $W^{\Gamma}(n \omega)$ are finite-dimensional.

Proof. Proposition 4.15 implies that $W^{\Gamma}(n \omega)_{\mu} \neq 0$ only if $\mu \in n \omega-Q_{0}^{+}$, and suppose that

$$
W^{\Gamma}(n \omega) \cong \bigoplus_{\mu \in P_{0}^{+}} V(\mu)^{n_{\mu}}
$$

is the decomposition of $W^{\Gamma}(n \omega)$ into irreducible $\mathfrak{g}_{0}$-modules. Note that the number of elements in $P_{0}^{+}$with the property $\mu \in n \omega-Q_{0}^{+}$is finite. The corollary follows if we prove that $\operatorname{dim} W^{\Gamma}(n \omega)_{\mu}<\infty$, since this implies $n_{\mu}<\infty$. That the dimension can't be infinity is a direct consequence of Corollary 7.3 .

As in the other cases we show that the Weyl modules are in connection with certain associated graded modules:

Proposition 7.5. Let $n \in \mathbb{N}$ such that $n=2 k$ if $n$ is even and $n=2 k-1$ if $n$ is odd. Then we get a surjective map, respectively an isomorphism, of $U\left(\mathfrak{C}\left(A_{2}^{(2)}\right)\right)$-modules

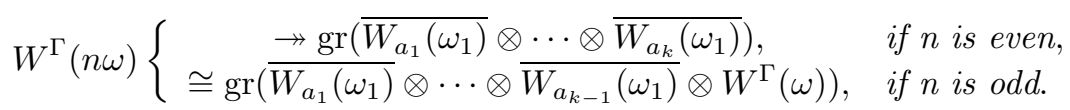

The map is given by $w_{n} \mapsto \underbrace{w_{\omega_{1}} \otimes \cdots \otimes w_{\omega_{1}}}_{k}$ if $n$ is even and $w_{n} \mapsto \underbrace{w_{\omega_{1}} \otimes \cdots \otimes w_{\omega_{1}}}_{k-1}$ $\otimes w_{\omega}$ otherwise.

Remark 7.6. We will proof the isomorphism claimed in the odd case in Section 7.2 and recall that the surjectivity of the maps in Proposition 7.5 follows by weight reasons.

Corollary 7.7. We obtain

$$
\operatorname{dim} W^{\Gamma}(n \omega) \geq\left\{\begin{array}{cl}
3^{\frac{n}{2}}, & \text { if } n \text { is even } \\
3^{\left\lceil\frac{n}{2}\right\rceil} 2, & \text { if } n \text { is odd. }
\end{array}\right.
$$

In Corollary 7.3 we proved that we can explicitly specify an integer such that the elements with higher powers of $t$ act by zero. Next we will refute the question as to whether there exists a smaller integer with the same property. To show this one can use the help of associated graded modules defined in Section 6 and Proposition 7.5 .

Lemma 7.8. Let $n \in \mathbb{N}$ as in Corollary 7.3. Then we have

$$
\left\{\begin{array}{cl}
\left(X_{\alpha, 0}^{-} \otimes t^{2 r}\right) w_{n} \neq 0,\left(X_{\alpha, 1}^{-} \otimes t^{2 r+1}\right) w_{n} \neq 0, & \text { for all } r<\frac{k}{2} \text { if } k \text { is even }, \\
\left(X_{\alpha, 0}^{-} \otimes t^{2 r}\right) w_{n} \neq 0,\left(X_{\alpha, 1}^{-} \otimes t^{2 s+1}\right) w_{n} \neq 0, & \text { for all } r<\frac{k+1}{2}, s<\frac{k-1}{2} \text { if } k \text { is odd }, \\
\left(X_{2 \alpha, 1}^{-} \otimes t^{2 r+1}\right) w_{n} \neq 0, & \text { if } r<k-1 .
\end{array}\right.
$$


Before we are in the position to prove our main result of this section we will formulate another necessary proposition:

Proposition 7.9. Let $n \in \mathbb{N}$ as in Corollary 17.3. Then we have surjective homomorphisms

$$
\begin{gathered}
W^{\Gamma}((n-2) \omega) \rightarrow\left\{\begin{array}{l}
U\left(\mathfrak{C}\left(A_{2}^{(2)}\right)\right)\left(X_{\alpha, 1}^{-} \otimes t^{k-1}\right) w_{n}, \quad \text { if } k \text { is even }, \\
U\left(\mathfrak{C}\left(A_{2}^{(2)}\right)\right)\left(X_{\alpha, 0}^{-} \otimes t^{k-1}\right) w_{n} \quad \text { if } k \text { is odd },
\end{array}\right. \\
W^{\Gamma}((n-4) \omega) \rightarrow U\left(\mathfrak{C}\left(A_{2}^{(2)}\right)\right)\left(X_{2 \alpha, 1}^{-} \otimes t^{2 k-3}\right) w_{n} .
\end{gathered}
$$

Proof. The proof is straightforward with Corollary 7.3 .

\subsection{Proof of Theorem 7.1.}

Proof. Note that Proposition 7.5 is a direct consequence of Theorem 7.1 and the Demazure character formula (see Theorem 4.6), since this provides

$$
\operatorname{dim} W^{\Gamma}(n \omega)=\operatorname{dim} V_{s_{1} t_{(n-1) \omega}}\left(\Lambda_{1}\right)=\operatorname{dim} V_{\left(s_{1} s_{0}\right)^{\left\lceil\frac{n}{2}\right\rceil} s_{1}}\left(\Lambda_{1}\right)=3^{\left\lceil\frac{n}{2}\right\rceil} 2 .
$$

We already know by Corollary 4.17 that the Demazure module $D(1 / 2, n \omega)$ is a quotient of the Weyl module $W^{\Gamma}(n \omega)$. So by Corollary 4.9 it remains to show that the following relations hold:

$$
\begin{gathered}
\left(X_{\alpha, 0}^{-} \otimes t^{2 r}\right)^{\max \{0, n-4 r\}+1} w_{n}=0, \\
\left(X_{\alpha, 1}^{-} \otimes t^{2 r+1}\right)^{\max \{0, n-2(2 r+1)\}+1} w_{n}=0, \\
\left(X_{2 \alpha, 1}^{-} \otimes t^{2 r+1}\right)^{\max \{0, k-r-1\}+1} w_{n}=0 .
\end{gathered}
$$

By Corollary 7.3 we can assume that the maximums are non-zero and further suppose that $\left(X_{\alpha, 0}^{-} \otimes t^{2 r}\right)^{n-4 r+1} w_{n} \neq 0$; hence $W^{\Gamma}(n \omega)_{(n-2(n-4 r+1)) \omega}[2 r(n-4 r+$ $1)] \neq 0$. By Proposition 7.9 and Proposition 4.15 (1) we get that

$$
W^{\Gamma}(n \omega)_{(n-2 j) \omega}[l]=0
$$

for all $l$ with

$$
l>\left\{\begin{array}{cl}
(k-1)+\cdots+(k-j)=j k-\frac{j(j+1)}{2}, & \text { if } 0 \leq j \leq k, \\
(k-1)+\cdots+(k-(n-j))=(n-j) k-\frac{(n-j)((n-j)+1)}{2}, & \text { if } k<j \leq n .
\end{array}\right.
$$

Hence,

$$
2 r(n-4 r+1) \leq\left\{\begin{array}{cl}
j k-\frac{j(j+1)}{2}, & \text { if } 0 \leq j \leq k, \\
(n-j) k-\frac{(n-j)((n-j)+1)}{2}, & \text { if } k<j \leq n
\end{array}\right.
$$

with $j=(n-4 r+1)$, which contradicts $2 r<k$. Exactly the same argumentation also shows (7.4) and (7.5).

Remark 7.10. An inspection of the proof of Theorem 7.1 shows that the condition that $n$ is odd is not needed. Thus the relations (7.3), (7.4), (17.5) also hold in $W^{\Gamma}(2 k \omega)$, but it is easy to see that they are not enough. For instance, in $W^{\Gamma}(6 \omega)$ we already have $\left(X_{\alpha, 0}^{-} \otimes t^{2}\right)^{2} w_{6}=0$, while relation (7.3) gives $\left(X_{\alpha, 0}^{-} \otimes t^{2}\right)^{3} w_{6}=0$. 


\section{ACKNOWLEDGEMENTS}

We would like to thank Vyjayanthi Chari and Peter Littelmann for helpful discussions. We would also like to thank the Hausdorff Research Institute for Mathematics and the organizers of the Trimester Program on the Interaction of Representation Theory with Geometry and Combinatorics, during which the ideas in the current paper were developed. The first author was partially sponsored by the DFG-Schwerpunktprogramm 1388 "Darstellungstheorie" and the second author by the "SFB/TR 12-Symmetries and Universality in Mesoscopic Systems".

\section{REFERENCES}

1. Jonathan Beck and Hiraku Nakajima, Crystal bases and two-sided cells of quantum affine algebras, Duke Math. J. 123 (2004), no. 2, 335-402. MR2066942 (2005e:17020)

2. Roger Carter, Lie algebras of finite and affine type, Cambridge Studies in Advanced Mathematics, vol. 96, Cambridge University Press, Cambridge, 2005. MR2188930 (2006i:17001)

3. Vyjayanthi Chari, On the fermionic formula and the Kirillov-Reshetikhin conjecture, Internat. Math. Res. Notices (2001), no. 12, 629-654. MR.1836791(2002i:17019)

4. Vyjayanthi Chari, Ghislain Fourier, and Tanusree Khandai, A categorical approach to Weyl modules, Transform. Groups 15 (2010), no. 3, 517-549. MR2718936

5. Vyjayanthi Chari, Ghislain Fourier, and Prasad Senesi, Weyl modules for the twisted loop algebras, J. Algebra 319 (2008), no. 12, 5016-5038. MR2423816 (2009e:17018)

6. Vyjayanthi Chari and Sergei Loktev, Weyl, Demazure and fusion modules for the current algebra of $\mathfrak{s l}_{r+1}$, Adv. Math. 207 (2006), no. 2, 928-960. MR2271991(2008a:17029)

7. Vyjayanthi Chari and Adriano Moura, The restricted Kirillov-Reshetikhin modules for the current and twisted current algebras, Comm. Math. Phys. 266 (2006), no. 2, 431-454. MR2238884 (2007m:17035)

8. Vyjayanthi Chari and Andrew Pressley, Integrable and Weyl modules for quantum affine $\mathrm{sl}_{2}$, Quantum groups and Lie theory (Durham, 1999), London Math. Soc. Lecture Note Ser., vol. 290, Cambridge Univ. Press, Cambridge, 2001, pp. 48-62. MR1903959 (2004c:17026)

9. Weyl modules for classical and quantum affine algebras, Represent. Theory $\mathbf{5}$ (2001), 191-223 (electronic). MR.1850556 (2002g:17027)

10. Michel Demazure, Une nouvelle formule des caractères, Bull. Sci. Math. (2) 98 (1974), no. 3, 163-172. MR0430001 (55:3009)

11. Boris Feigin and Sergei Loktev, On generalized Kostka polynomials and the quantum Verlinde rule, Differential topology, infinite-dimensional Lie algebras, and applications, Amer. Math. Soc. Transl. Ser. 2, vol. 194, Amer. Math. Soc., Providence, RI, 1999, pp. 61-79. MR.1729359 (2002b:17007)

12. Multi-dimensional Weyl modules and symmetric functions, Comm. Math. Phys. 251 (2004), no. 3, 427-445. MR2102326 (2005m:17005)

13. Ghislain Fourier, Tanusree Khandai, Deniz Kus, and Alistair Savage, Local weyl modules for equivariant map algebras with free abelian group actions, Journal of Algebra, Volume 350, Issue 1, 15 January 2012, Pages 386-404. MR2859894

14. Ghislain Fourier and Peter Littelmann, Tensor product structure of affine Demazure modules and limit constructions, Nagoya Math. J. 182 (2006), 171-198. MR.2235341 (2007e:17021)

15. __ Weyl modules, Demazure modules, KR-modules, crystals, fusion products and limit constructions, Adv. Math. 211 (2007), no. 2, 566-593. MR2323538(2008k:17005)

16. Ghislain Fourier, Nathan Manning, and Prasad Senesi, Global Weyl modules for the twisted loop algebras, Abh. Math. Semin. Univ. Hambg. 85 (2013), 53-82. MR3055822

17. William Fulton and Joe Harris, Representation theory, Graduate Texts in Mathematics, vol. 129, Springer-Verlag, New York, 1991, A first course, Readings in Mathematics. MR.1153249 (93a:20069)

18. Goro Hatayama, Atsuo Kuniba, Masato Okado, Taichiro Takagi, and Zengo Tsuboi, Paths, crystals and fermionic formulae, MathPhys odyssey, 2001, Prog. Math. Phys., vol. 23, Birkhäuser Boston, Boston, MA, 2002, pp. 205-272. MR.1903978 (2003e:17020)

19. Victor G. Kac, Infinite-dimensional Lie algebras, third ed., Cambridge University Press, Cambridge, 1990. MR1104219 (92k:17038) 
20. Michael Steven Kleber, Finite dimensional representations of quantum affine algebras, ProQuest LLC, Ann Arbor, MI, 1998, Thesis (Ph.D.)-University of California, Berkeley. MR2697975

21. Shrawan Kumar, Kac-Moody groups, their flag varieties and representation theory, Progress in Mathematics, vol. 204, Birkhäuser Boston Inc., Boston, MA, 2002. MR1923198(2003k:22022)

22. Michael Lau, Representations of multiloop algebras, Pacific J. Math. 245 (2010), no. 1, 167184. MR 2602688

23. Olivier Mathieu, Construction du groupe de Kac-Moody et applications, C. R. Acad. Sci. Paris Sér. I Math. 306 (1988), no. 5, 227-230. MR932325(89e:17013)

24. Hiraku Nakajima, Quiver varieties and finite-dimensional representations of quantum affine algebras, J. Amer. Math. Soc. 14 (2001), no. 1, 145-238. MR.1808477 (2002i:17023)

25. Katsuyuki Naoi, Weyl modules, Demazure modules and finite crystals for non-simply laced type, arXiv:1012.5480.

26. Erhard Neher, Alistair Savage, and Prasad Senesi, Irreducible finite-dimensional representations of equivariant map algebras, Trans. Amer. Math. Soc. 364 (2012), no. 5, 2619-2646. $\operatorname{MR} 2888222$

Mathematisches Institut, Universität Zu Köln, 50931 KÖln, Germany

E-mail address: gfourier@math.uni-koeln.de

Mathematisches Institut, Universität Zu Köln, 50931 Köln, Germany

E-mail address: dkus@math.uni-koeln.de 